\title{
CRASHES, VOLATILITY, AND THE EQUITY PREMIUM: LESSONS FROM S\&P 500 OPTIONS
}

\author{
Pedro Santa-Clara and Shu Yan*
}

Abstract-We use a novel pricing model to imply time series of diffusive volatility and jump intensity from S\&P 500 index options. These two measures capture the ex ante risk assessed by investors. Using a simple general equilibrium model, we translate the implied measures of ex ante risk into an ex ante risk premium. The average premium that compensates the investor for the ex ante risks is $70 \%$ higher than the premium for realized volatility. The equity premium implied from option prices is shown to significantly predict subsequent stock market returns.

\section{Introduction}

$\mathrm{T}$ HIS paper uses option prices to estimate the risk of the stock market as it is perceived ex ante by investors. We consider two types of risk in stock prices: diffusion risk and jump risk. ${ }^{1}$ As Merton (1980) argued, diffusion risk can be accurately measured from the quadratic variation of the realized price process. In contrast, since even highprobability jumps may fail to materialize in sample, the ex ante jump risk perceived by investors may be quite different from the ex post realized variation in prices. Therefore, studying measures of realized volatility and realized jumps from the time series of stock prices will give us a limited picture of the risks investors fear. Fortunately, since options are priced on the basis of ex ante risks, they give us a privileged view of the risks perceived by investors. Using option data solves the "peso problem" in measuring jump risk from realized stock returns.

In our model, both the volatility of the diffusion shocks and the intensity of the jumps vary over time following separate stochastic processes. ${ }^{2}$ Our model is quadratic in the state variables. This allows the covariance structure of the shocks to the state variables to be unrestricted, which proves

Received for publication January 19, 2007. Revision accepted for publication July 29, 2008.

* Santa Clara: Universidade Nova de Lisboa and NBER; Yan: University of South Carolina.

We thank Julio Rotemberg (the editor), two anonymous referees, Ravi Bansal, David Bates, Michael Brandt, Michael Brennan, João Cocco, Marcelo Fernandes, Mikhail Chernov, Christopher Jones, Jun Liu, Francis Longstaff, Jun Pan, Alessio Saretto, and Bill Schwert for helpful comments. We also thank seminar participants at Instituto de Empresa (Madrid), Universidade Nova de Lisboa, University of Arizona, University of South Carolina, University of Vienna, the Fifteenth FEA Conference at USC, the NBER Spring 2008 Asset Pricing Meeting, the 2008 LusoBrazilian Finance Meeting, and the University of Amsterdam Fourth Annual Empirical Asset Pricing Retreat. An appendix with derivations of some expressions in the paper and some additional results is available online at http://www.mitpressjournals.org/doi/suppl/10.1162/rest.2010. 11549 .

${ }^{1}$ There is ample empirical evidence for this kind of specification. See, for example, Jorion (1988), Bakshi, Cao, and Chen (1997), and Bates (2000).

${ }^{2}$ In contrast, other jump-diffusion models impose a constant jump intensity (Merton, 1976; Bates, 1996) or make it a deterministic function of the diffusive volatility (Bates, 2000; Duffie, Pan, \& Singleton, 2000 Pan, 2002). The empirical analysis shows that the jump intensity varies a lot and that although it is related to the diffusive volatility, it has its own source of shocks. to be important in the empirical analysis. We are still able to solve for the European option prices in a manner similar to the affine case of Duffie, Pan, and Singleton (2000). In the empirical application, the model is shown to produce pricing errors of the order of magnitude of the bid-ask spread in option prices.

When we calibrate the model to S\&P 500 index option prices from the beginning of 1996 to the end of 2002, we obtain time series of the implied diffusive volatility and jump intensity. We find that the innovations to the two risk processes are not very correlated with each other, although both are negatively correlated with stock returns. The two components of risk vary substantially over time and show a high degree of persistence. The diffusive volatility process varies between close to 0 and $36 \%$ per year, which is in line with the level of ex post risk measured from the time series of stock returns. The jump intensity process shows even wider variation. Sometimes the probability of a jump is 0 , while at other times it is more than $99 \%{ }^{3}$ We estimate that the expected jump size is $-9.8 \%$. Interestingly, we do not observe any such large jumps in the time series of the S\&P 500 index in our sample, not even around the times when the implied jump intensity is very high. These were therefore cases in which the jumps that were feared did not materialize. However, the perceived risks are still likely to have affected the expected return in the stock market at those times.

To investigate the impact of ex ante risk on expected returns, we solve for the stock market risk premium in a simple economy with a representative investor with power utility for final wealth. We find that the equilibrium risk premium is a function of both the stochastic volatility and the jump intensity. Given the implied stochastic volatility and jump intensity processes, together with the estimated coefficient of risk aversion for the representative investor, we estimate the time series of the ex ante equity premium. This is the expected excess return demanded by the investor to hold the entire wealth in the stock market when facing the diffusion and jump risks implicit in option prices. We decompose the ex ante equity premium into compensation for diffusive risk and compensation for jump risk. We find the ex ante equity premium to be quite variable over time. In our sample, the equity premium demanded by the representative investor varies from as low as $0.3 \%$ and as high as $54.9 \%$ per year! The compensation for jump risk is on

\footnotetext{
${ }^{3}$ We calculate this probability as $1-e^{-\lambda}$, where $\lambda$ is the instantaneous jump intensity. This calculation assumes that the jump intensity remains constant for an entire year. Since the process we estimate for the jump intensity is strongly mean reverting, this figure overstates the probability of a jump during the year.
} 
average more than half of the total premium. Moreover, in times of crisis, the jump risk commands a premium of $45.4 \%$ per year and can be close to $100 \%$ of the total premium. ${ }^{4}$

The ex ante premium evaluated at the average levels of diffusive volatility and jump intensity implied from the options in our sample is $11.8 \%$. In contrast, the same investor would require a premium of only $6.8 \%$ as compensation for the realized volatility (i.e., the sample standard deviation of returns) during the same sample period. Therefore, the required compensation for the ex ante risks is more than $70 \%$ higher than the compensation for the realized risks! This finding supports the peso explanation of the equity premium puzzle proposed by Rietz (1988), Brown, Goetzmann, and Ross (1995), and Barro (2006).

According to this explanation, there is a risk of a substantial crash in the stock market that has not materialized in the sample but justifies a larger risk premium than what has traditionally been thought reasonable along the lines of Mehra and Prescott (1985).

To show that the equity premium implied from the options market is indeed related to stock prices, we run predictive regressions of stock returns on the lagged implied equity premium. We find that the regression coefficient is significant for different predictability horizons. For onemonth returns, the $R^{2}$ is $4.1 \%$, and it becomes $6.6 \%$ for three-month returns. The regression coefficient is close to 1 for the three-month horizon as expected for an unbiased forecast. Finally, we examine the relation between the option implied equity premium and three variables related to financial crises: the T-bill rate, the spread of bank commercial paper over T-bills, and the spread of high-yield bonds over Treasuries. Intuitively, the jump risk we uncover in options should be related to large-scale financial crises in which the Fed lowers interest rates, interbank loans dry up and become more expensive, and corporations are more likely to default. We find significant relations between these variables and the implied equity premium, with an $R^{2}$ as high as $13.9 \%$ for the high-yield spread.

The paper closest to ours is Pan (2002). ${ }^{5}$ She estimates a jump-diffusion model from both the time series of the S\&P 500 index and its options from 1989 to 1996 . She uses the pricing model proposed by Bates (2000) which has a square root process for the diffusive variance and jump intensity proportional to the diffusive variance. The jump risk premium is specified to be linear in the variance. Pan finds a significant jump premium of roughly $3.5 \%$, which is of the same order of magnitude of the volatility risk premium of

\footnotetext{
4 This variation in the equity premium is extreme and may be due to overfitting a particular equilibrium model. It also assumes that option prices reflect accurately investors' expectations. These potential limitations are further discussed in section VB.

5 Other related work includes Ait-Sahalia, Wang, and Yared (2001), Bates (2001), Bliss and Panigirtzoglou (2004), Chernov and Ghysels (2000), Engle and Rosenberg (2002), Eraker (2004), and Jackwerth (2000).
}

5.5\%. The main difference between our paper and hers is that in Pan's framework, it is hard to disentangle the diffusion and jump risks and risk premia since they are all driven by a single state variable, the diffusive volatility.

Finally, a word of caution. Our analysis relies on option prices, and, of course, options may be systematically mispriced. That would bias our ex ante risk measures. Coval and Shumway (2001) and Driessen and Maenhout (2003) report empirical evidence that some option strategies have unusually high Sharpe ratios, which may indicate mispricing. Santa-Clara and Saretto (2004) show that transaction costs and margin requirements impose substantial limits to arbitrage in option markets, which may allow mispricings to persist.

The paper proceeds as follows. In section II, we present the dynamics of the stock market index under the objective and the risk-adjusted probability measures, and we derive an option pricing formula. In section III, we discuss the data and the econometric approach. The model estimates and its performance in pricing the options in the sample are covered in section IV. Section V contains the main results of the paper, the analysis of the risks implied from option prices and what they imply for the equity premium. Section VI concludes.

\section{The Model}

In this section we introduce a new model of the dynamics of the stock market return that displays stochastic diffusive volatility and jumps with stochastic intensity. We derive the equilibrium stock market risk premium in a simple economy with a representative investor with CRRA utility. This risk premium compensates the investor for both volatility and jump risks. We also obtain the risk-adjusted dynamics of the stock, volatility, and jump intensity processes and use them to price European options.

\section{A. Stock Market Dynamics}

We model the dynamics of the stock market index with two sources of risk: diffusive risk, captured by a Brownian motion, and jump risk, modeled as a Poisson process. The diffusive volatility and the intensity of the jump arrivals are stochastic and interdependent. We parameterize the processes as:

$$
\begin{aligned}
& d S=\left(r+\phi-\lambda \mu_{Q}\right) S d t+Y S d W_{S}+Q S d N \\
& d Y=\left(\mu_{Y}+\kappa_{Y} Y\right) d t+\sigma_{Y} d W_{Y} \\
& d Z=\left(\mu_{Z}+\kappa_{Z} Z\right) d t+\sigma_{Z} d W_{Z} \\
& \ln (1+Q) \sim \mathcal{N}\left(\ln \left(1+\mu_{Q}\right)-\frac{1}{2} \sigma_{Q}^{2}, \sigma_{Q}^{2}\right) \\
& \operatorname{Prob}(d N=1)=\lambda d t, \quad \text { where } \lambda=Z^{2}
\end{aligned}
$$




$$
\Sigma=\left(\begin{array}{ccc}
1 & \rho_{S Y} & \rho_{S Z} \\
\rho_{S Y} & 1 & \rho_{Y Z} \\
\rho_{S Z} & \rho_{Y Z} & 1
\end{array}\right)
$$

$W_{S}, W_{Y}$, and $W_{Z}$ are Brownian motions with constant correlation matrix $\Sigma$, and $N$ is a Poisson process with arrival intensity $\lambda . Q$ is the percentage jump size and is assumed to follow a displaced log-normal distribution independently over time. This guarantees that the jump size cannot be less than -1 and therefore that the stock price remains positive at all times. We assume that $N$ and $Q$ are independent of each other and that $Q$ is independent of the Brownian motions. The instantaneous variance of the stock return is $V=Y^{2} . r$ is the risk-free interest rate, assumed constant for convenience. We also assume that the stock pays no dividends, although it would be trivial to accommodate them by adding a term in the drift of the stock price. $\phi$ is the risk premium on the stock, which we show below to be a function of $Y$ and $Z$. Finally, the term $\lambda \mu_{Q}$ adjusts the drift for the average jump size.

In our model, the stock price, the stochastic volatility, and the jump intensity follow a joint quadratic jump-diffusion process where the stochastic processes of $V$ and $\lambda$ are the squares of linear (gaussian) processes of $Y$ and $Z$, respectively. ${ }^{6}$ Applying Ito's lemma, we can write the processes followed by $V$ and $\lambda$ :

$$
\begin{aligned}
& d V=\left(\sigma_{Y}^{2}+2 \mu_{Y} Y+2 \kappa_{Y} Y^{2}\right) d t+2 \sigma_{Y} Y d W_{Y} \\
& d \lambda=\left(\sigma_{Z}^{2}+2 \mu_{Z} Z+2 \kappa_{Z} Z^{2}\right) d t+2 \sigma_{Z} Z d W_{Z} .
\end{aligned}
$$

The drift and diffusion terms in equations (7) and (8) depend on the signs of the gaussian state variables $Y$ and $Z$. Note that the instantaneous correlation between $d S$ and $d V$ is constant, $\rho_{S Y}$, while the instantaneous correlation between $d S$ and $d Y$ is $\operatorname{sgn}(Y) \rho_{S Y}$ where sgn $(\cdot)$ is the sign function, since $\sqrt{V}=\operatorname{sgn}(Y) Y$ and $\sqrt{\lambda}=\operatorname{sgn}(Z) Z .^{7}$

Without the jump component, our model collapses to a stochastic volatility model similar to that of Stein and Stein (1991). ${ }^{8}$ It can easily be seen that the model does not belong to the affine family of Duffie et al. (2000), in that the drifts

\footnotetext{
${ }^{6}$ Cheng and Scaillet (2007) also study quadratic option pricing models. Ahn, Dittmar, and Gallant (2002), Chen, Filipovic, and Poor (2004), and Leippold and $\mathrm{Wu}$ (2002) present quadratic models of the term structure.

${ }^{7}$ In our model, the correlation between $d S$ and $d \lambda$, as well as the correlation between $d V$ and $d \lambda$ can change signs, whereas the correlation between $d S$ and $d V$ always has the sign of $\rho_{S Y}$. The negative correlation between $d S$ and $d V$ is well documented in the literature as the leverage effect. This gives us a strong prior on the sign of $\rho_{S Y}$. However, our intuition about the signs of the other two correlations and whether they should or should not change over time is much weaker. Our specification allows the correlations to be freely estimated without having to make assumptions about their signs and even allowing the signs to change over time. In the empirical sections, we estimate this model and find that $Y$ and $Z$ end up taking negative values (very close to 0 in all cases) in only 4 out of the 366 weeks of our sample. Therefore, there is little evidence of changing signs in the correlations between the state variables.

${ }^{8}$ In Stein and Stein (1991), $\sqrt{V}$ follows an Ornstein-Uhlenbeck process, whereas in our model, $V=Y^{2}$ with $Y$ following an Ornstein-Uhlenbeck process. Since the square root function is not globally invertible, the two
}

and the covariance terms in $V$ and $\lambda$ are not linear in the state variables. For instance, the covariance between $d V$ and $d \lambda$ is $\rho_{Y} \sigma_{Y} \sigma_{Z} Y Z$.

Our model belongs to the family of linear-quadratic jump-diffusion models. It is the first model in which the jump intensity $\lambda$ follows explicitly its own stochastic process. In contrast, existing jump diffusion models either assume that the jump intensity is constant or make it a deterministic function of other state variables such as stochastic volatility. ${ }^{9}$ For instance, Pan (2002) assumes that $\lambda$ is a linear function of $V$. It is of course an empirical issue whether the jump intensity is completely driven by volatility or whether it has its own separate source of uncertainty. The empirical sections shed some light on this matter.

We do not include jumps in volatility as do Eraker, Johannes, and Polson (2003) and Broadie, Chernov, and Johannes (2007). After a large movement in stock prices, other large movements are likely to follow. To capture this feature of the data with stochastic volatility alone, in a model with no jumps or with only independent and identically distributed (i.i.d.) jumps, volatility needs to jump up (and stay up) following the large movement in the stock. In our model, the clustering of large movements is captured by an increase in jump intensity (instead of a jump in volatility), after which jumps tend to cluster together. ${ }^{10}$

We now turn our attention to finding the risk premium $\phi$. Consider a representative investor who has wealth $W$ and allocates it entirely to the stock market. ${ }^{11}$ For simplicity, we assume that there is no intermediate consumption, so the investor chooses an optimal portfolio to maximize the utility of terminal wealth:

$$
\max _{w} \mathrm{E}_{t}\left[u\left(W_{T}, T\right)\right]
$$

where $\mathrm{E}_{t}(\cdot)$ is the conditional expectation operator, $w$ is the fraction of wealth invested in the stock, $T$ is the terminal date, and $u$ is the utility function. Define the value function of the investor as

$$
J\left(W_{t}, Y_{t}, Z_{t}, t\right) \equiv \max _{w} \mathrm{E}_{t}\left[u\left(W_{T}, T\right)\right] .
$$

Following Merton (1973) and using subscripts to denote the partial derivative of $J$, a solution to equation (9) satisfies the Bellman equation,

$$
0=\max _{w}\left[J_{t}+\mathscr{L}(J)\right]
$$

are not the same. See also Ball and Roma (1994) and Schobel and Zhu (1999).

${ }^{9}$ Some of these models can be transformed to allow the jump intensity to evolve separately from the volatility. For example, the two-factor jump-diffusion model in Bates (2000) admits such a transform for extreme values of one of the state variables and for some model parameters.

${ }^{10}$ Although we do not have a formal analysis, it does not seem easy to identify a model with jumps in volatility and time-varying jump intensity.

${ }^{11}$ Naik and Lee (1990) offer a related general equilibrium model for pricing options. 
with

$$
\begin{aligned}
\mathscr{L}(J)= & W J_{W}\left(r+w \phi-w \lambda \mu_{Q}\right)+J_{Y}\left(\mu_{Y}+\kappa_{Y} Y\right) \\
& +J_{Z}\left(\mu_{Z}+\kappa_{Z} Z\right)+\frac{1}{2} w^{2} W^{2} J_{W W} Y^{2} \\
& +\frac{1}{2} J_{Y Y} \sigma_{Y}^{2}+\frac{1}{2} J_{Z Z} \sigma_{Z}^{2}+w W J_{W Y} \rho_{S Y} \sigma_{Y} Y \\
& +w W J_{W Z} \rho_{S Z} \sigma_{Z} Y+J_{Y Z} \rho_{Y Z} \sigma_{Y} \sigma_{Z}+Z^{2} \mathrm{E}_{Q}[\Delta J],
\end{aligned}
$$

where $\mathrm{E}_{Q}(\cdot)$ is the expectation with respect to the distribution of $Q$. The term $\Delta J \equiv J(W(1+w Q), Y, Z, t)-J(W$, $Y, Z, t)$ captures jumps in the value function. In equilibrium, the risk-free asset is in zero net supply. Therefore, the representative investor holds all the wealth in the stock market, that is, $w=1$. Differentiating equation (10) with respect to $w$ and substituting in $w=1$, we obtain the risk premium on the stock,

$$
\begin{aligned}
\phi= & -\frac{J_{W W}}{J_{W}} W Y^{2}-\rho_{S Y} \sigma_{Y} \frac{J_{W Y}}{J_{W}} Y-\rho_{S Z} \sigma_{Z} \frac{J_{W Z}}{J_{W}} Y \\
& -\mathrm{E}\left[\frac{\Delta J_{W}}{J_{W}} Q\right] Z^{2},
\end{aligned}
$$

where $\Delta J_{W} \equiv J_{W}(W(1+Q), Y, Z, t)-J_{W}(W, Y, Z, t)$. The stock risk premium contains four components: the variance of the marginal utility of wealth and the covariances of the marginal utility of wealth with the diffusive volatility, the jump intensity, and the jump size, respectively.

For tractability, we concentrate our attention on the case of power utility: $u=W_{T}^{1-\gamma} /(1-\gamma)$, where $\gamma>1$ is the constant relative risk aversion coefficient of the investor. In the online appendix, we show that the risk premium on the stock consistent with equilibrium in this economy is a function of $Y$ and $Z$ :

$$
\begin{aligned}
& \phi(Y, Z, \tau)=\gamma Y^{2}-\rho_{S Y} \sigma_{Y}\left(B_{Y}+2 C_{Y Y} Y+2 C_{Y Z} Z\right) Y \\
& -\rho_{S Z} \sigma_{Z}\left(B_{Z}+2 C_{Y Z} Y+2 C_{Z Z} Z\right) Y \\
& -\left[e^{-\gamma \ln \left(1+\mu_{Q}\right)+\frac{1}{2} \gamma(\gamma-1) \sigma_{Q}^{2}}\left(1+\mu_{Q}-e^{\gamma \sigma_{Q}^{2}}\right)-\mu_{Q}\right] Z^{2} \\
& =\gamma Y^{2}-\left(\begin{array}{ll}
\rho_{S Y} \sigma_{Y} & \left.\rho_{S Z} \sigma_{Z}\right) B Y
\end{array}\right. \\
& -2\left(\rho_{S Y} \sigma_{Y} \quad \rho_{S Z} \sigma_{Z}\right)\left(\begin{array}{c}
C_{Y Y} \\
C_{Y Z}
\end{array}\right) Y^{2} \\
& -2\left(\begin{array}{ll}
\rho_{S Y} \sigma_{Y} & \rho_{S Z} \sigma_{Z}
\end{array}\right)\left(\begin{array}{l}
C_{Y Z} \\
C_{Z Z}
\end{array}\right) Y Z \\
& -\left[e^{-\gamma \ln \left(1+\mu_{Q}\right)+\frac{1}{2} \gamma(\gamma-1) \sigma_{Q}^{2}}\left(1+\mu_{Q}-e^{\gamma \sigma_{Q}^{2}}\right)-\mu_{Q}\right] Z^{2},
\end{aligned}
$$

where we define $\tau \equiv T-t, B(\tau)=\left(\begin{array}{c}B_{Y} \\ B_{Z}\end{array}\right)$ is a $2 \times 1$ matrix function, and $C(\tau)=\left({ }_{C_{Y Z} C_{Y Z}}^{C_{Y Z}}\right)$ is a $2 \times 2$ symmetric matrix function. $B$ and $C$ solve the following system of ODEs with the initial conditions $B(0)=\left(\begin{array}{l}0 \\ 0\end{array}\right)$ and $C(0)=\left(\begin{array}{ll}0 & 0 \\ 0 & 0\end{array}\right)$ :

$$
\begin{aligned}
& B^{\prime}=\left(\Lambda^{\top}+2 C \Gamma\right) B+2 C \Pi \\
& C^{\prime}=\Theta+C \Lambda+\Lambda^{\top} C+2 C \Gamma C,
\end{aligned}
$$

where ${ }^{\top}$ denotes the transpose of a matrix (or the complex transpose in the case of a complex matrix), and the constant matrices $\Theta, \Pi, \Lambda$, and $\Gamma$ are defined as

$$
\begin{aligned}
& \Theta \equiv\left(\begin{array}{cc}
-\frac{1}{2} \gamma(\gamma-1) & 0 \\
0 & e^{-\gamma \ln \left(1+\mu_{Q}\right)+\frac{1}{2} \gamma(\gamma-1) \sigma_{Q}^{2}} \\
& {\left[\gamma\left(1+\mu_{Q}\right)-(\gamma-1) e^{\gamma \sigma_{Q}^{2}}\right]-1}
\end{array}\right) \\
& \Pi \equiv\left(\begin{array}{c}
\mu_{Y} \\
\mu_{Z}
\end{array}\right) \\
& \Lambda \equiv\left(\begin{array}{cc}
\kappa_{Y} & 0 \\
0 & \kappa_{Z}
\end{array}\right) \\
& \Gamma \equiv\left(\begin{array}{cc}
\sigma_{Y}^{2} & \rho_{Y Z} \sigma_{Y} \sigma_{Z} \\
\rho_{Y Z} \sigma_{Y} \sigma_{Z} & \sigma_{Z}^{2}
\end{array}\right) .
\end{aligned}
$$

For a given value of the risk-aversion coefficient $\gamma$, the ODEs (14)-(15) can be quickly solved numerically. In the special case where there is no stochastic volatility and jumps, the equity premium (12) collapses to the first term, $\gamma Y^{2}=\gamma V$, as shown by Merton (1973). In the special case where there is no stochastic volatility and the jump intensity is constant, equation (12) collapses to the first term and the last term. The other two terms in equation (12) involving $B$ and $C$ capture the effects of shifting investment opportunities when both $Y$ and $Z$ are stochastic. The first three terms in equation (13) involve $Y$ only and thus correspond to compensation for stochastic volatility, and the last term compensates the investor for jump risk as it involves $Z$ only. The interaction between the volatility and jump intensity risks is captured by the cross term involving $Y Z$.

In related work, Liu and Pan (2003) derive the optimal portfolio of a CRRA investor who can hold the stock, an option on the stock, and a risk-free asset. In their model, the stock market has stochastic diffusive volatility and jumps of deterministic size with the jump intensity driven by the stochastic volatility. In contrast to our paper, theirs is a partial equilibrium analysis that takes the price of risk as given.

\section{B. Option Pricing}

We can price European options in this economy. In the online appendix we show that the risk-adjusted dynamics of the stock price can be written as: ${ }^{12}$

\footnotetext{
12 The stock price should be interpreted as being ex-dividend since we are interested in pricing options that are not dividend protected.
} 


$$
\begin{aligned}
& d S=\left(r-\lambda^{*} \mu_{Q}^{*}\right) S d t+Y S d W_{S}^{*}+Q^{*} S d N^{*} \\
& d Y=\left(\mu_{Y}^{*}+\kappa_{Y Y}^{*} Y+\kappa_{Y Z}^{*} Z^{*}\right) d t+\sigma_{Y} d W_{Y}^{*} \\
& d Z^{*}=\left(\mu_{Z}^{*}+\kappa_{Z Y}^{*} Y+\kappa_{Z Z}^{*} Z^{*}\right) d t+\sigma_{Z}^{*} d W_{\lambda}^{*} \\
& \ln \left(1+Q^{*}\right) \sim \mathcal{N}\left(\ln \left(1+\mu_{Q}^{*}\right)-\frac{1}{2} \sigma_{Q}^{2}, \sigma_{Q}^{2}\right) \\
& \operatorname{Prob}\left(d N^{*}=1\right)=\lambda \lambda^{*} d t, \quad \text { where } \lambda^{*}=Z^{* 2} \\
& \Sigma=\left(\begin{array}{ccc}
1 & \rho_{S Y} & \rho_{S Z} \\
\rho_{S Y} & 1 & \rho_{Y Z} \\
\rho_{S Z} & \rho_{Y Z} & 1
\end{array}\right)
\end{aligned}
$$

with the following simple relations between the model parameters under the objective and risk-adjusted probability measures: $:^{13}$

$$
\begin{aligned}
& \left(\begin{array}{l}
\mu_{Y}^{*} \\
\mu_{Z}^{*}
\end{array}\right)=\left(\begin{array}{ll}
1 & 0 \\
0 & b
\end{array}\right)(\Pi+\Gamma B) \\
& \left(\begin{array}{cc}
\kappa_{Y Y}^{*} & \kappa_{Y Z}^{*} \\
\kappa_{Z Y}^{*} & \kappa_{Z Z}^{*}
\end{array}\right)=\left(\begin{array}{cc}
1 & 1 / b \\
b & 1
\end{array}\right) \circ[\Lambda-\gamma \\
& \left.\quad \times\left(\begin{array}{ll}
\rho_{S Y} \sigma_{Y} & 0 \\
\rho_{S Z} \sigma_{Z} & 0
\end{array}\right)+2 \Gamma C\right] \\
& \sigma_{Z}^{*}=b \sigma_{Z} \\
& Z^{*}=b Z \\
& \mu_{Q}^{*}=\left(1+\mu_{Q}\right) e^{-\gamma \sigma_{Q}^{2}-1} \\
& b=\left(1+\mu_{Q}\right)^{-\frac{1}{2} \gamma} e^{\frac{1}{4} \gamma(\gamma+1) \sigma_{Q}^{2}}
\end{aligned}
$$

where $\Pi, \Lambda$, and $\Gamma$ are defined as before, and $\circ$ is the element-by-element product of two matrices. The riskadjusted coefficients on the left-hand sides of the equations above are related to the coefficients under the objective probability measure by the risk-aversion coefficient $\gamma$. Note that the compensation for the jump risk is reflected in the changed jump intensity as well as the changed distribution of the jump size, whereas the compensation for the diffusive risk requires only a change in the drift of the processes. ${ }^{14}$

In contrast to the complete market setting of Black and Scholes (1973), the added random jump sizes make the market incomplete with respect to the risk-free asset, the underlying stock, and any finite number of option contracts. Consequently the change of probability is not unique. We use the equilibrium pricing condition from the endowment

\footnotetext{
${ }^{13}$ Note that $Y$ and $Z^{*}$ now appear in the drift terms of each other, while $Y$ and $Z$ do not under the objective probability measure.

${ }_{14}$ Note that in general, all the parameters governing the jump process may change when the probability measure changes. However, in the case of a representative investor with power utility function, the volatility of jump size $\sigma_{Q}$ does not change.
}

economy with a CRRA representative investor to identify the change of probability measure. It turns out that this particular change of probability measure involves changing the jump size and intensity.

Following the approach of Lewis (2000), we find the price $f$ of a European call option with strike price $K$ and maturity date $T:{ }^{15}$

$$
\begin{gathered}
f\left(S, Y, Z^{*}, t ; K, T\right)=S-\frac{e^{-r \tau}}{2 \pi} \int_{\frac{i}{2}-\infty}^{\frac{i}{2}+\infty} \frac{K^{i k+1}}{k^{2}-i k} \\
\times e^{-i k(r \tau+\ln S)+A^{*}(\tau)+B^{*}(\tau)^{\top} U^{*}+U^{* \top} C^{*}(\tau) U^{*}} d k,
\end{gathered}
$$

where $i=\sqrt{-1}, k$ is the integration variable, $U^{*} \equiv\left({ }_{Z^{*}}^{Y}\right)$, $A^{*}(\tau)$ is a scalar function, $B^{*}(\tau)=\left(\begin{array}{c}B_{Y}^{*} \\ B_{Z}^{*}\end{array}\right)$ is a $2 \times 1$ matrix function, and $C^{*}(\tau)=\left(\begin{array}{l}C_{Y Z}^{*} C_{Y Z}^{*} \\ C_{Y Z}^{*} C_{Z Z}^{*}\end{array}\right)$ is a $2 \times 2$ symmetric matrix function. $A^{*}, B^{*}$, and $C^{*}$ solve the following system of ODEs with initial conditions $A^{*}(0)=0, B^{*}(0)=\left(\begin{array}{l}0 \\ 0\end{array}\right)$, and $C^{*}(0)=\left(\begin{array}{ll}0 & 0 \\ 0 & 0\end{array}\right)$ :

$$
\begin{aligned}
& A^{*^{\prime}}=\Pi^{* \top} B^{*}+\frac{1}{2} B^{* \top} \Gamma^{*} B^{*}+\operatorname{tr}\left(\Gamma^{*} C^{*}\right) \\
& B^{* \prime}=\left(\Lambda^{* \top}+2 C^{*} \Gamma^{*}\right) B^{*}+2 C^{*} \Pi^{*} \\
& C^{* \prime}=\Theta^{*}+C^{*} \Lambda^{*}+\Lambda^{* \top} C^{*}+2 C^{*} \Gamma^{*} C^{*},
\end{aligned}
$$

where $\operatorname{tr}(\cdot)$ is the trace of a matrix, and the matrices $\Theta^{*}, \Pi^{*}$, $\Lambda^{*}$, and $\Gamma^{*}$ are defined as

$$
\begin{aligned}
& \Theta * \equiv\left(\begin{array}{cc}
-\frac{1}{2}\left(k^{2}-i k\right) & 0 \\
0 & i k \mu_{Q}^{*}+e^{-i k \ln \left(1+\mu_{Q}^{*}\right)-\frac{1}{2}\left(k^{2}-i k\right) \sigma_{Q}^{2}-1}
\end{array}\right) \\
& \Pi^{*} \equiv\left(\begin{array}{l}
\mu_{Y}^{*} \\
\mu_{Z}^{*}
\end{array}\right) \\
& \Lambda^{*} \equiv\left(\begin{array}{cc}
\kappa_{Y Y}^{*}-i k \rho_{S Y} \sigma_{Y} & \kappa_{Y Z}^{*} \\
\kappa_{Z Y}^{*}-i k \rho_{S Z} \sigma_{Z}^{*} & \kappa_{Z Z}^{*}
\end{array}\right) \\
& \Gamma^{*} \equiv\left(\begin{array}{cc}
\sigma_{Y}^{2} & \rho_{Y Z} \sigma_{Y} \sigma_{Z}^{*} \\
\rho_{Y Z} \sigma_{Y} \sigma_{Z}^{*} & \sigma_{Z}^{* 2}
\end{array}\right) .
\end{aligned}
$$

This formula involves the inverse Fourier transform of an exponential of a quadratic form of the state variables, $Y$ and $Z^{*}$. The ODEs that define $A^{*}, B^{*}$, and $C^{*}$ can be easily solved numerically. Again, the online appendix presents the gruesome algebra.

\section{Estimation}

In this section we discuss the data and the econometric method used to estimate the model and imply the time series of diffusive volatility and jump intensity.

\footnotetext{
${ }^{15}$ Although it contains a complex integral, the result is real.
} 
Table 1.-Implied Volatilities of S\&P 500 Index Options

\begin{tabular}{cccc}
\hline \hline \multirow{2}{*}{ Moneyness } & \multicolumn{3}{c}{ Days to Expiration } \\
\cline { 2 - 4 }$S / K<0.95$ & $T \leq 45$ & $45<T \leq 90$ & $T>90$ \\
\hline \multirow{2}{*}{$0.95 \leq S / K<1.05$} & 23.83 & 21.39 & 20.7 \\
& $(4.67)$ & $(4.14)$ & $(3.88)$ \\
& {$[193]$} & {$[262]$} & {$[466]$} \\
$S / K \geq 1.05$ & $(4.95)$ & 20.75 & 21.32 \\
& {$[3,155]$} & $(4.62)$ & $(4.26)$ \\
& 27.43 & {$[2,928]$} & {$[2,211]$} \\
& $(5.67)$ & 25.54 & 24.3 \\
& {$[2,029]$} & $(5.22)$ & $(4.63)$ \\
& Note: We report average implied volatility, the standard deviation of implied volatilities (in parenthe-
\end{tabular}

viation of implied volatilities (in parentheses), and the number of options (in brackets) within each moneyness-maturity bucket.

\section{A. Data}

For our econometric analysis, we use the European S\&P 500 index options traded on the Chicago Board Options Exchange (CBOE) for the period January 1996 to December 2002 obtained from OptionMetrics. The S\&P 500 index and its dividends are obtained from Datastream. The interest rates are LIBOR (middle) rates also obtained from Datastream.

Since the stocks within the S\&P 500 index pay dividends, whereas our model does not account for payouts, we adjust the index level by the expected future dividends in order to compute the option prices. Realized dividends are used as a proxy for expected dividends. The dividend-adjusted stock price corresponding to the maturity of a given option is calculated by subtracting the present value of the future realized dividends until the maturity of the option from the current index level. Interest rates are interpolated to match the maturities of the options.

We estimate our model at weekly frequency. We collect the index level, interest rates, and option prices on Wednesday of each week. ${ }^{16}$ To ensure that the options we use are liquid enough, we choose contracts with maturity shorter than a year and moneyness between 0.85 and 1.15. We exclude options with no trading volume and options with open interest of fewer than 100 contracts. We use only put options in our study as they are more liquid than call options and since using both option types would be redundant given put-call parity. For each contract, we use the average of the bid and ask prices as the value of the option. We exclude options with time to maturity fewer than 10 days and prices less than $\$ 1 / 8$ to mitigate market microstructure problems. Finally, we check for no-arbitrage violations in option prices. We end up with 366 trading days and 14,416 option prices in our sample, or roughly 40 options per day.

Table 1 reports the average implied volatility of the options in the sample. Rather than tabulating the option prices, we show the Black-Scholes implied volatilities since

\footnotetext{
${ }^{16}$ If Wednesday is not a trading day, we obtain prices from, in order of preference, Tuesday, Thursday, Monday, or Friday.
}

Figure 1.-Time Series of ImPlied Volatilities
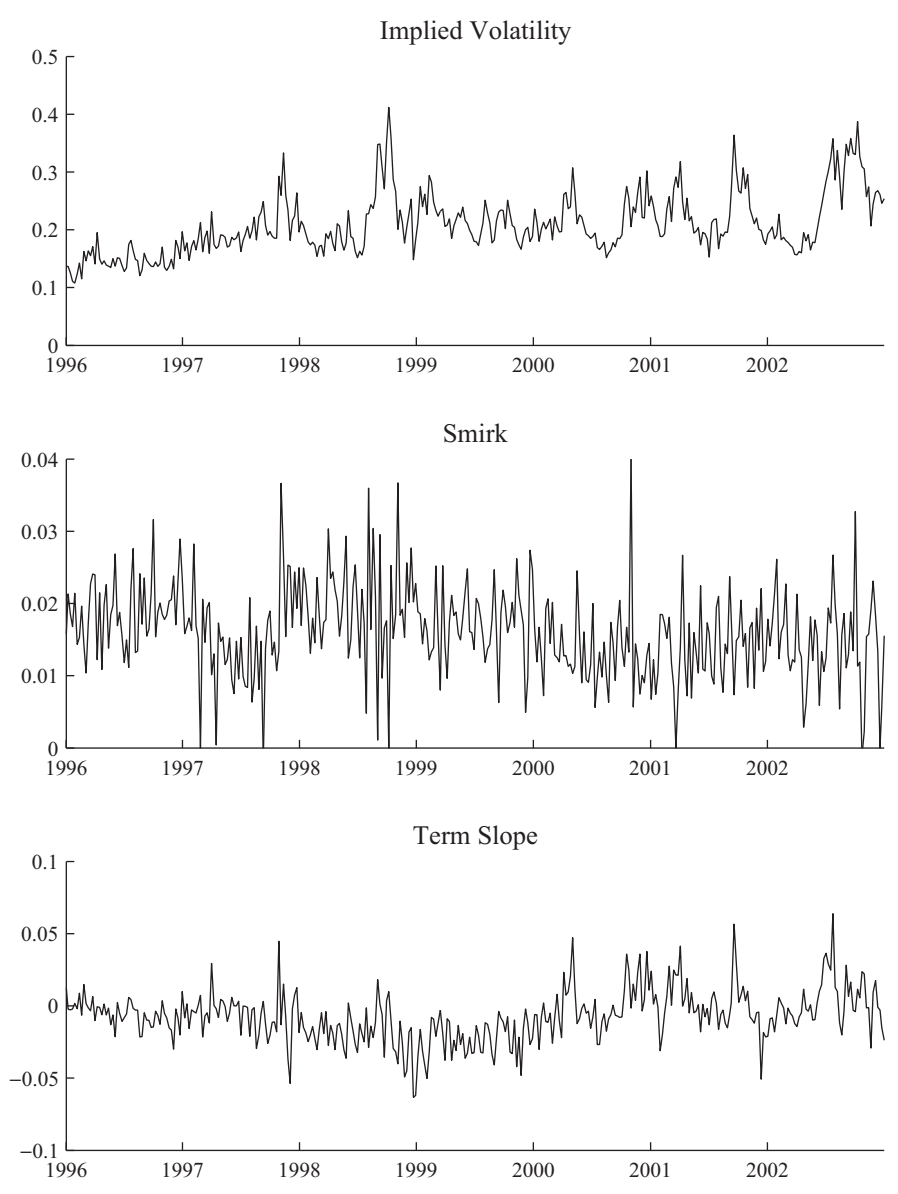

they are easier to interpret. ${ }^{17}$ We divide all options into nine buckets according to moneyness (stock price divided by the strike price) and time to maturity: moneyness less than 0.95 , between 0.95 and 1.05 , and above 1.05; time to maturity less than 45 days, between 45 and 90 days, and greater than 90 days. Note that when moneyness is greater than 1 , the put options are out of the money. The average implied volatility across all options in our sample was $22.77 \%$. The first panel of figure 1 plots the time series of the implied volatility of the short-term (maturity less than 45 days and as close as possible to 30 days) option with moneyness closest to $S / K=1$ (at-the-money). We can see that the implied volatility changes substantially over time. The spike in the implied volatilities observed in fall 1998 corresponds to the Russian default crisis and long-term capital management debacle. For a fixed maturity, we can observe that the implied volatilities decrease and then increase with the strike price. This is the well-known "volatility smirk." The second panel plots the time series of the "smirk," defined as

\footnotetext{
${ }^{17}$ Here, we use the Black-Scholes model to invert option prices for implied volatilities. This does not mean that the options are priced in the market according to that model and, indeed, we will use our model with stochastic volatility and jumps to price the options in the empirical section below. The Black-Scholes formula is used as a device to translate option prices into volatilities, which are easier to interpret.
} 
the difference between the Black-Scholes implied volatilities of two short-term put options with moneyness closest to $S / K=1.025$ (out-of-the-money) and $S / K=1$ (at-themoney), respectively. It shows that the smirk is positive all the time and there are changes in the steepness of the smirk over time. The third panel of figure 1 plots the time series of the "term slope," defined as the difference between the Black-Scholes implied volatilities of the two at-the-money put options with two maturities: short term (defined as above) and long term (greater than 45 days and as close as possible to 60 days), respectively. It shows that there is some variation in the slope of the term structure through time. During our sample period, the term slope was on average close to flat.

\section{B. Econometric Method}

We adopt an implied-state quasi-maximum likelihood (IS-QML) estimation method that is similar to the impliedstate generalized method of moments (IS-GMM) of Pan (2002). Our approach combines information from stock and option prices, taking advantage of the existence of an analytical option pricing formula. In Pan (2002), volatility is the only latent state variable that has to be implied. We extend Pan's method to our setting, where both volatility and jump intensity are latent and have to be implied. We estimate the model parameters by maximizing the joint likelihood function of a discrete approximation of the continuous time transition densities of the state variables and the density of the cross-sectional option pricing errors. One advantage of the QML method is that we do not need to choose the moment conditions, which is always a sensitive choice in GMM.

Our modeling of the quasi-likelihood function is inspired by Duffee (2002), who estimates a dynamic term structure model. We assume that some options are observed without error or imply the state variables, while others are observed with error. Our quasi-likelihood function combines the time series distribution of the implied state variables and the cross-sectional distribution of the pricing errors. In contrast, Pan (2002) uses only the time-series data of the implied state variables to define her moment conditions.

For estimation, we use weekly data for the stock index and four put option contracts $\left\{S_{t}, P_{t}^{1}, P_{t}^{2}, P_{t}^{3}, P_{t}^{4}\right\}$, where $P_{t}^{1}$ and $P_{t}^{2}$ have the shortest maturity and $P_{t}^{3}$ and $P_{t}^{4}$ have the second shortest maturity. $P_{t}^{1}$ and $P_{t}^{3}$ are closest to at-themoney; $P_{t}^{2}$ and $P_{t}^{4}$ are closest to moneyness $(S / K)$ of 1.05 . The maturity of the first two options is greater than 15 days and as close as possible to 30 days, while the maturity of the last two options is greater than 45 days and as close as possible to 60 days. ${ }^{18}$ All four contracts are actively traded.

\footnotetext{
${ }^{18}$ Using two maturities helps in identifying $Y$ and $Z$ since jumps and stochastic volatility have different effects on short- and long-term options. We are constrained in using options with longer maturities than a few months since they are not liquid. The results are robust to choosing options with moneyness $0.95,0.975$, or 1.025 .
}

We use $P_{t}^{1}$ and $P_{t}^{2}$ to imply the state variables $Y_{t}$ and $Z_{t}$ and use $P_{t}^{3}$ and $P_{t}^{4}$ to compute the pricing errors.

Note that the $i$ th put option price can be expressed as $P_{t}^{i}=f\left(S_{t}, Y_{t}, Z_{t} ; K_{i}, T_{i}, \theta\right)$, where $f(\cdot)$ is given by equation (28) together with put-call parity, $K_{i}$ and $T_{i}$ are the strike price and time to maturity of the $i$ th option, and $\theta=\left(\mu_{Y}, \kappa_{Y}, \sigma_{Y}, \mu_{Z}, \kappa_{Z}, \sigma_{Z}, \mu_{Q}, \sigma_{Q}, \rho_{S Y}, \rho_{S Z}, \rho_{Y Z}, \gamma\right)$ is the vector of model parameters under the objective probability measure. Given $\theta$, proxies $Y_{t}^{\theta}$ and $Z_{t}^{\theta}$ for the unobserved $Y_{t}$ and $Z_{t}$ can be obtained by inverting the system of equations $P_{t}^{1}=f\left(S_{t}, Y_{t}^{\theta}, Z_{t}^{\theta} ; K_{1}, T_{1}, \theta\right)$ and $P_{t}^{2}=f\left(S_{t}, Y_{t}^{\theta}, Z_{t}^{\theta} ; K_{2}, T_{2}, \theta\right){ }^{19}$

Given $Y_{t}^{\theta}$ and $Z_{t}^{\theta}$, the model-based option prices $P_{t}^{3, \theta}$ and $P_{t}^{4, \theta}$ for the third and fourth options can be calculated using the option pricing formula. We then compute the BlackScholes implied volatilities $\sigma_{t}^{3, \theta}$ and $\sigma_{t}^{4, \theta}$ for these two options based on the model prices. The measurement errors are defined as $\epsilon_{t}^{i, \theta}=\sigma_{t}^{i, \theta}-\sigma_{t}^{i}$, where $i=3$ and 4, and $\sigma_{t}^{i}$ is the Black-Scholes implied volatility of the $i$ th option based on the observed market price. Let $\epsilon_{t}^{\theta}=\left(\begin{array}{c}\epsilon_{t}^{3, \theta} \\ \epsilon_{t}^{4, \theta}\end{array}\right)$ denote the vector of measurement errors.

For week $t$, the log likelihood under the objective probability measure is defined as

$$
l_{t}(\theta)=\log f_{X}\left(X_{t}^{\theta} \mid X_{t-1}^{\theta}\right)+\log f_{\epsilon}\left(\epsilon_{t}^{\theta}\right),
$$

where $f_{X}(\cdot)$ is the conditional density of the vector of state variables $X_{t}^{\theta}=\left(S_{t}, Y_{t}^{\theta}, Z_{t}^{\theta}\right)^{\top}$ and $f_{\epsilon}(\cdot)$ is the density function of the vector of pricing errors $\epsilon_{t}^{\theta}$. This specification implicitly assumes that the pricing errors are independent of the state variables.

Generalizing the approach of Ball and Torous (1983), we use the truncated Poisson-normal mixture distribution to approximate $f_{X}(\cdot)$ for the jump-diffusion model in equations (1) to (3). Let $\Delta t$ be the time interval of discretization, which is $1 / 52$ for our weekly frequency data. We approximate equations (1) to (3) by the following discrete system:

$$
\Delta \ln S_{t}=\left(r+\phi_{t-1}-\lambda_{t-1} \mu_{Q}\right) \Delta t+Y_{t-1} \sqrt{\Delta t} \epsilon_{S, t}+Q_{t} B_{t}
$$

$$
\begin{aligned}
& \Delta Y_{t}=\left(\mu_{Y}+\kappa_{Y} Y_{t-1}\right) \Delta t+\sigma_{Y} \sqrt{\Delta t} \epsilon_{Y, t} \\
& \Delta Z_{t}=\left(\mu_{Z}+\kappa_{Z} Z_{t-1}\right) \Delta t+\sigma_{Z} \sqrt{\Delta t} \epsilon_{Z, t},
\end{aligned}
$$

where $\left(\epsilon_{S, t}, \epsilon_{Y, t}, \epsilon_{Z, t}\right) \sim$ i.i.d. $\mathcal{N}(0, \Sigma), Q_{t} \sim$ i.i.d. $\mathcal{N}\left(\mu_{Q}\right.$, $\left.\sigma_{Q}^{2}\right), Q_{t}$ and $\epsilon_{t}$ are independent, $B_{t} \sim$ i.i.d. $\mathscr{P}\left(\lambda_{t-1} \Delta t\right)$ where $\mathscr{P}(\cdot)$ is the truncated Poisson distribution with truncation taken at $M$, the maximum number of jumps that may occur during a time interval. ${ }^{20}$ We fix $M$ to be 5 in our paper.

\footnotetext{
${ }^{19}$ It is not always the case that $Y$ and $Z$ can be inverted for a given vector of parameters $\theta$. The intuition is that bivariate quadratic equations do not always have real solutions. We impose the constraint that the vector of parameters $\theta$ allows inversion of $Y$ and $Z$.

${ }^{20}$ The density function of $\mathscr{P}(\lambda \Delta t)$ is proportional to $f(x)=$ $e^{-\lambda \Delta t}(\lambda \Delta t)^{x} / x$ ! for $x=0,1,2, \ldots, M$.
} 
Our discrete model, equations (32) to (34), allows multiple (up to $M$ ) jumps in a time interval, while Ball and Torous (1983) consider at most only one jump during a time interval. We approximate $f_{X}(\cdot)$ by the likelihood function of equations (32) to (34), which is a mixture of truncated Poisson and normal distributions. ${ }^{21}$ We examine the precision of the approximation in the online appendix.

To model $f_{\epsilon}(\cdot)$, we assume that the option pricing error vector $\epsilon_{t}^{\theta}$ has an i.i.d. bivariate normal distribution with constant covariance matrix. Given the definition of the $\log$-likelihood function $l_{t}(\theta)$, the QML parameter vector $\theta$ is obtained from the optimization program:

$$
\max _{\theta} L(\theta)=\max _{\theta} \sum_{t=1}^{T} l_{t}(\theta) .
$$

We employ an optimization algorithm similar to that of Duffee (2002). In step 1, we generate starting values for the parameter vector $\theta$. In step 2, we use the formula (28) and option prices $P_{t}^{1}, P_{t}^{2}$ to derive the implied state variables $Y_{t}^{\theta}$ and $Z_{t}^{\theta}$. In step 3, we use the nonlinear Simplex algorithm to obtain a new parameter vector that improves the QML value. We then repeat the above steps until convergence is achieved. The standard errors of the parameter estimates are obtained from the last QML optimization step. The estimation time for the SV-SJ model ranges from two to four hours depending on the choice of the initial parameter values.

In addition to the general model (SV-SJ), we also estimate two restricted cases: the stochastic volatility model (SV) and the constant jump intensity model (SV-J). For the restricted models, volatility is the only latent state variable that needs to be implied. Therefore, in those cases, we invert just the short-term at-the-money option $P_{t}^{1}$ to imply the state variable $Y_{t}$.

It is important to point out that the options are priced under the risk-adjusted probability measure, while the transition densities of the state variables are specified under the objective probability measure. The fact that the likelihood function combines information from both the objective and the risk-adjusted distribution of the state variables has a crucial role in the estimation of the risk-aversion parameter $\gamma$. A necessary identification condition is that the transformation between the objective and the risk-adjusted probability measure be monotonic in terms of $\gamma$. In our framework, this transformation is given by equations (22) to (27), which depends on $\gamma$. For the SV model, the difference between $\kappa_{Y Y}^{*}$ and $\kappa_{Y}$ is $-\gamma \rho_{S Y} \sigma_{Y}$, which is clearly monotonic in $\gamma \cdot{ }^{22}$ For the SV-SJ model, the difference between

\footnotetext{
${ }^{21}$ It is well known that the log-likelihood function for a mixture of normal distributions is unbounded, but it is still possible to obtain consistent and asymptotically normal distributed estimates by constraining the maximum likelihood algorithm (see, for example, Hamilton, 1994).

${ }^{22} \mathrm{In}$ some studies of the $\mathrm{SV}$ models $-\gamma \rho_{S Y} \sigma_{Y}$ is called the market price of risk. Using the parameter estimates reported in table 2, this market price of risk is positive.
}

Table 2.-Estimated Parameters

\begin{tabular}{cccc}
\hline \hline & SV & SV-J & SV-SJ \\
\hline$\mu_{Y}$ & 1.929 & 1.756 & 2.841 \\
& $(0.340)$ & $(0.314)$ & $(0.346)$ \\
$\kappa_{Y}$ & -9.201 & -9.218 & -18.079 \\
& $(1.541)$ & $(1.545)$ & $(2.065)$ \\
$\sigma_{Y}$ & 0.306 & 0.315 & 0.334 \\
& $(0.008)$ & $(0.009)$ & $(0.010)$ \\
$\mu_{Z}$ & & & 7.745 \\
& & & $(1.396)$ \\
$\kappa_{Z}$ & & & -9.436 \\
$\sigma_{Z}$ & & & $(1.567)$ \\
& & & 1.529 \\
$\mu_{Q}$ & & -0.070 & $-0.045)$ \\
& & $(0.012)$ & $(0.013)$ \\
$\sigma_{Q}$ & & 0.283 & 0.160 \\
& & $(0.013)$ & $(0.013)$ \\
$\rho_{S Y}$ & -0.731 & -0.728 & -0.495 \\
& $(0.014)$ & $(0.014)$ & $(0.025)$ \\
$\rho_{S Z}$ & & & -0.597 \\
& & & $(0.022)$ \\
$\rho_{Y Z}$ & & & 0.168 \\
$\gamma$ & 1.977 & 1.984 & $(0.045)$ \\
$\gamma$ & $(0.391)$ & $(0.369)$ & $(0.352)$ \\
$\operatorname{RMSE}(\%)$ & 3.348 & 2.730 & 2.131 \\
\hline
\end{tabular}

Note: We report the standard errors in parentheses under the estimated parameters.

$\kappa_{Y Y}^{*}$ and $\kappa_{Y}$ is more complex but can still be shown to be monotonic in $\gamma$ using the parameter estimates reported in table 2. Intuitively, the identification of $\gamma$ comes from the mean reversion speed observed in the implied state variables, coupled with the mean reversion speed implicit in the option prices. The mean reversion speeds of $Y$ and $Z$ under the objective probability measure enter the likelihood function through the transition density $f_{X}\left(X_{t}^{\theta} \mid X_{t-1}^{\theta}\right)$, while the same coefficients under the risk-neutral probability measure enter the likelihood function through the density of the pricing errors $f_{\epsilon}\left(\epsilon_{t}^{\theta}\right)$. Since the transformation between the mean reversion speeds under the two probability measures is monotonic, the QML algorithm finds a unique value of $\gamma$ that maximizes the combined likelihood function. ${ }^{23}$ The precision of the estimate of $\gamma$ is remarkable and much greater than could be achieved by estimating this parameter from the drift of the stock market alone.

Another potential problem in our QML approach is that the approximation of the conditional likelihood function $f_{X}(\cdot)$ by the truncated Poisson-normal mixture distribution may bias the estimates of the model parameters. In the online appendix, we conduct Monte Carlo simulations to verify the precision of the approximation. We show that the QML estimates are close to the true parameters (used for the simulations) indicating no significant bias in our estimation approach.

\footnotetext{
${ }^{23}$ We thank an anonymous referee for this explanation.
} 
TABle 3.- IMPlied Diffusive Volatility AND Jump INTENSITY

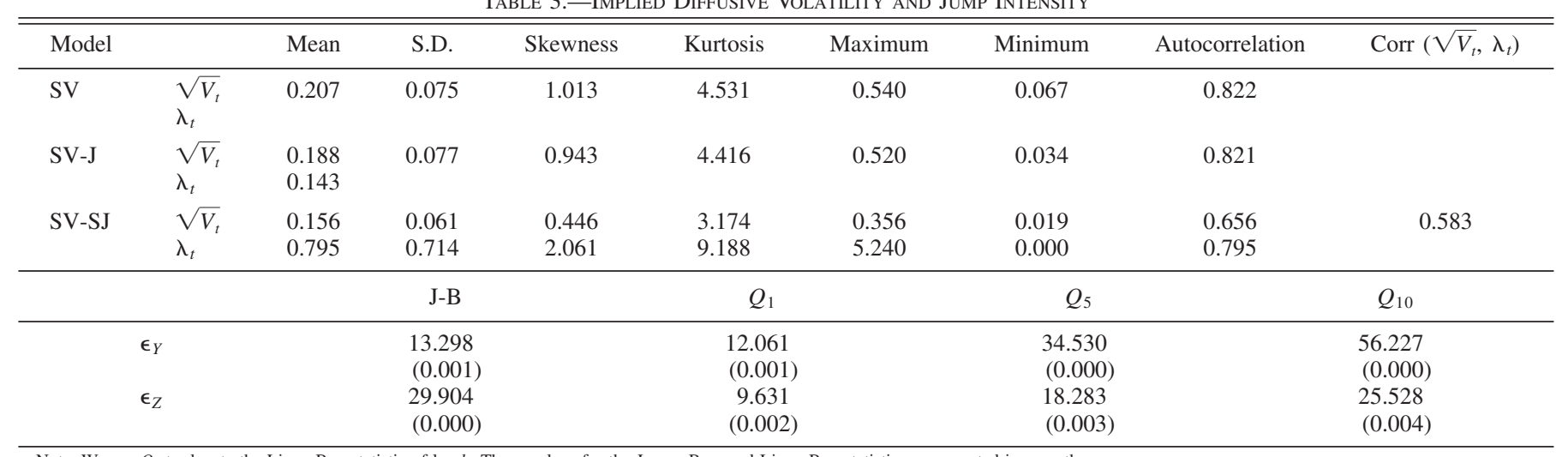

Note: We use $Q_{k}$ to denote the Ljung-Box statistic of lag $k$. The $p$-values for the Jarque-Bera and Ljung-Box statistics are reported in parentheses.

\section{Empirical Results}

In this section we discuss the empirical results. We present the model estimates and discuss the performance of the model in pricing options.

\section{A. Model Estimates}

The SV-SJ model of stochastic volatility and stochastic jump intensity contains the pure stochastic volatility model (SV) and the constant jump intensity model (SV-J) as special cases. In the SV model, we restrict $\mu_{Q}=\sigma_{Q}=$ $\mu_{Z}=\kappa_{Z}=\rho_{S Z}=\rho_{Y Z}=0$. In the SV-J model, we restrict $\mu_{Z}=\kappa_{Z}=\rho_{S Z}=\rho_{Y Z}=0$, and $\lambda_{t}=\bar{\lambda}$ is a constant. ${ }^{24}$

Table 2 reports the estimated parameters for the three models. We can compare to some extent the parameter estimates for the SV model with the estimates reported by Bakshi, Cao, and Chen (1997) and Pan (2002). However, notice that their SV model is the square root model of Heston (1993), whereas ours is similar to the model of Stein and Stein (1991). Also, their sample periods are different from ours. Bakshi et al. use S\&P 500 index options data from 1988 to 1991, and Pan uses S\&P 500 index options data from 1989 to 1996.

In Bakshi et al. (1997) and Pan (2002), the square root of the estimated long-run mean of $V$ is $18.7 \%$ and $11.7 \%$, respectively. Our estimate of the long-run mean of $\sqrt{V}(=|Y|)$, given by $\mu_{Y} / \kappa_{Y}$, is a bit higher, at $21.0 \%$. These differences are mainly due to the difference in sample periods. The estimates of mean-reversion speed are 1.15 and 7.10 in their papers, whereas it is 9.20 in our paper, implying stronger mean reversion. ${ }^{25}$ The volatility of volatility is 0.39 and 0.32 in their

\footnotetext{
${ }^{24}$ Note that option pricing formula for the SV-J model cannot be obtained from that of the SV-SJ model by restricting the corresponding parameters. A similar option pricing formula can be derived using the same approach as for the SV-SJ model.

${ }^{25}$ Our estimate of mean reversion speed is much faster than those of some early studies. One explanation is that previous studies generally use an early sample period. As a check, we examined the at-the-money nearest-to-maturity implied volatility for the period 1990-1995 (from CBOE data since Option Metrics is not available for that period). The first-order autocorrelation coefficients are 0.911 and 0.822 for the 1990
}

papers, and it is 0.31 in our paper. ${ }^{26}$ The correlation between the stock and volatility processes is estimated to be -0.64 and -0.57 in their papers, and it is -0.73 in our paper.

Bakshi et al. (1997) and Pan (2002) also estimate an SV-J model. In this case, the square root of their estimated long-run mean of $V$ is $18.7 \%$ and $11.6 \%$ in their papers, whereas our estimate is $19.0 \%$. The mean-reversion speed is estimated as 0.98 and 7.10 in their papers and 9.22 in our paper. The volatility of volatility is 0.42 and 0.28 in their papers and 0.31 in our estimate. The correlation between volatility and the stock is -0.76 and -0.52 in their papers and -0.73 in ours. Finally, they estimate the mean jump size to be -5 percent and -0.3 percent, respectively, whereas we estimate it to be -7 percent. In summary, our estimates for the restricted SV and SV-J models are comparable with the findings in other studies despite the differences in the data sets and models.

We next concentrate our attention on the SV-SJ model. All the coefficients of the model are significant at any conventional level of significance. Table 3 reports summary statistics for the implied time series of $\sqrt{V_{t}}$ and $\lambda_{t}$, which are plotted in figure 2 .

The average level of volatility is $15.6 \%$, and the average level of jump intensity-loosely speaking, the expected number of jumps over the next year-is 0.80 . The average jump size is $-9.8 \%$, which is $40 \%$ higher than the average jump size in the SV-J model.

Both the volatility and jump intensity series exhibit substantial variation through time. The diffusive volatility varies between $1.9 \%$ and $35.6 \%$. The jump intensity varies from less than 0.01 to over 5 during the 1998 financial crisis. Interestingly the two risk sources, although correlated, can display very different behavior: from times of high diffusive and jump risks, as in the second half of 2002;

1995 and 1996-2002 periods, respectively, indicating faster mean reversion in the more recent period.

${ }^{26}$ According to Ito's lemma, the volatility of volatility in Heston's model is half that in our model. So our estimate of volatility of volatility is about twice of those in Bakshi et al. (1997) and Pan (2002). But this higher volatility is offset by a much faster rate of mean reversion. 
Figure 2.-Implied Diffusive Volatility AND JumP Intensity
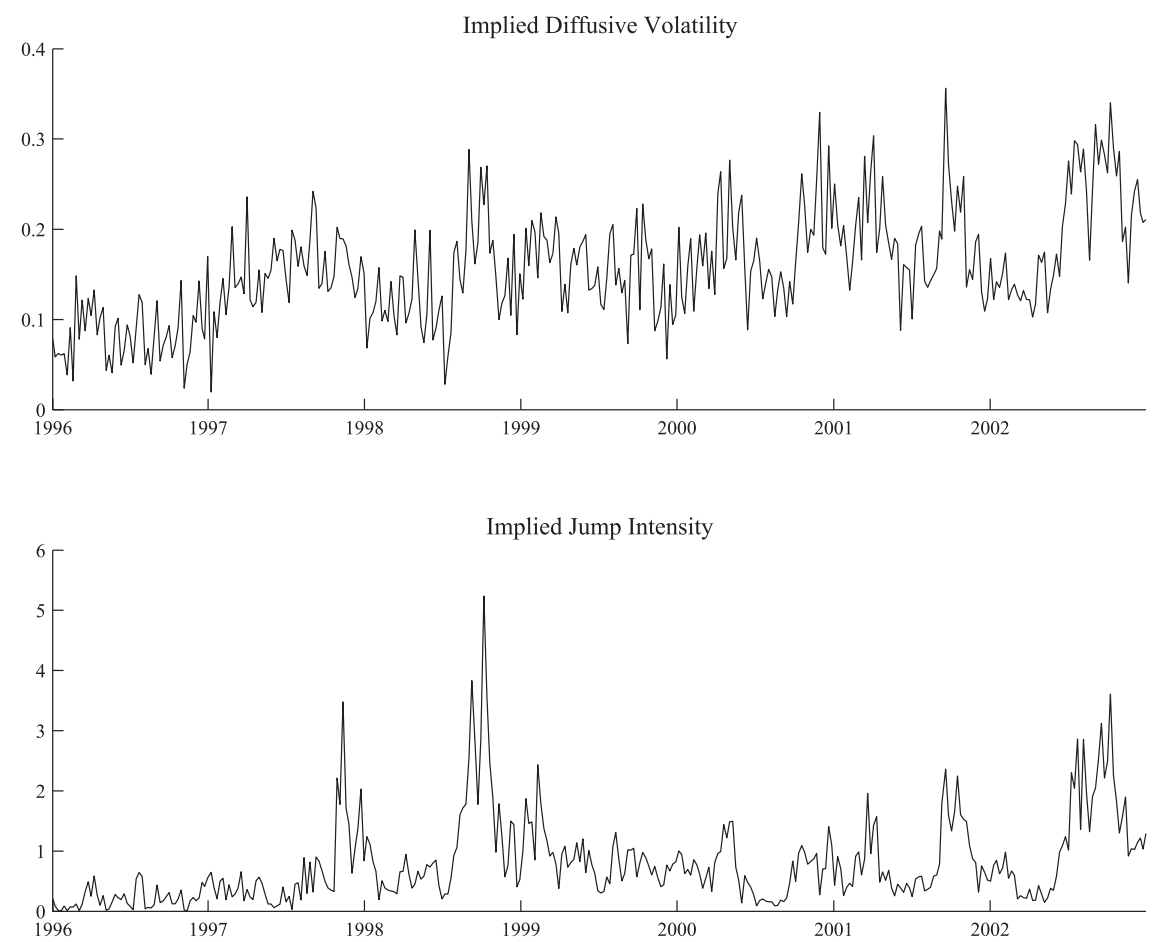

to times when jump risk is high but diffusive risk is low, as in the fall of 1998; to times when both risks are low, as in the beginning of 1996 .

The implied time series of volatility from the SV-SJ model is different from those of the other two models. The average implied volatility, $15.6 \%$, is much lower in the SV-SJ model than in the SV model since the stochastic volatility in the latter model needs to account for all the risk, including the jump risk.

The estimated volatility process in the SV-SJ model is mean reverting at about twice the speed as in the SV and SV-J models. The implied time series of stochastic volatility and jump intensity show autocorrelations of 0.66 and 0.79 , respectively.

The estimated correlation between the increments of the diffusive volatility and jump intensity is quite low, at 0.17 . This is evidence that the two processes are largely uncorrelated and do not support models that make jump intensity vary with the level of diffusive volatility. Increments of the diffusive volatility are negatively correlated with stock returns, -0.50 , which is smaller than that in the SV and SV-J models. Changes in jump intensity are also negatively correlated with stock returns at a higher absolute value, -0.60 .

Overall, our results are also consistent with the recent literature on multifactor variance models (Alizadeh, Brandt, \& Diebold, 2002; Chacko \& Viceira, 2003; Chernov et al., 2002; Engle \& Lee, 1999; Ghysels, Santa-Clara, \& Valkanov, 2005), which finds reliable support for the existence of two factors driving the conditional variance. The first factor is found to have high persistence and low volatility, whereas the second factor is transitory and highly volatile. The evidence from estimating jump diffusions with stochastic volatility points in a similar direction (Jorion, 1988; Anderson, Benzoni, \& Lund, 2002; Chernov et al., 2002; Eraker et al., 2003). For example, Chernov et al. (2002) show that the diffusive component is highly persistent and has low variance, whereas the jump component is by assumption not persistent and is highly variable.

The second panel of table 3 reports Jarque-Bera and LjungBox statistics for the innovations of the state variables $Y$ and $Z$. The Jarque-Bera statistics are significant, indicating nonnormality of the innovations. The Ljung-Box statistics are also significant, implying serial correlation in the innovations. Both diagnostic tests indicate misspecification of the SV-SJ model. Pan (2002) also finds that her jump-diffusion model is misspecified. Since her model is similar to the SV-J model in our paper, the sources of misspecification are likely to be similar. Pan argues that the misspecification shows evidence of jumps in volatility, as modeled in Duffie et al. (2000) and empirically studied in Eraker et al. (2003).

\section{B. Option Pricing Performance}

We evaluate the option pricing performance of the model in terms of the root mean squared error (RMSE) of BlackScholes implied volatilities. The implied volatility error of a given option is the difference between the implied volatilities calculated from market price and model price. Allowing the jump intensity to vary stochastically proves to be quite important for options pricing. As reported in the last row of table 2, the RMSE of the SV-SJ model for all options in our 
Figure 3.-Market and Fitted Implied Volatilities
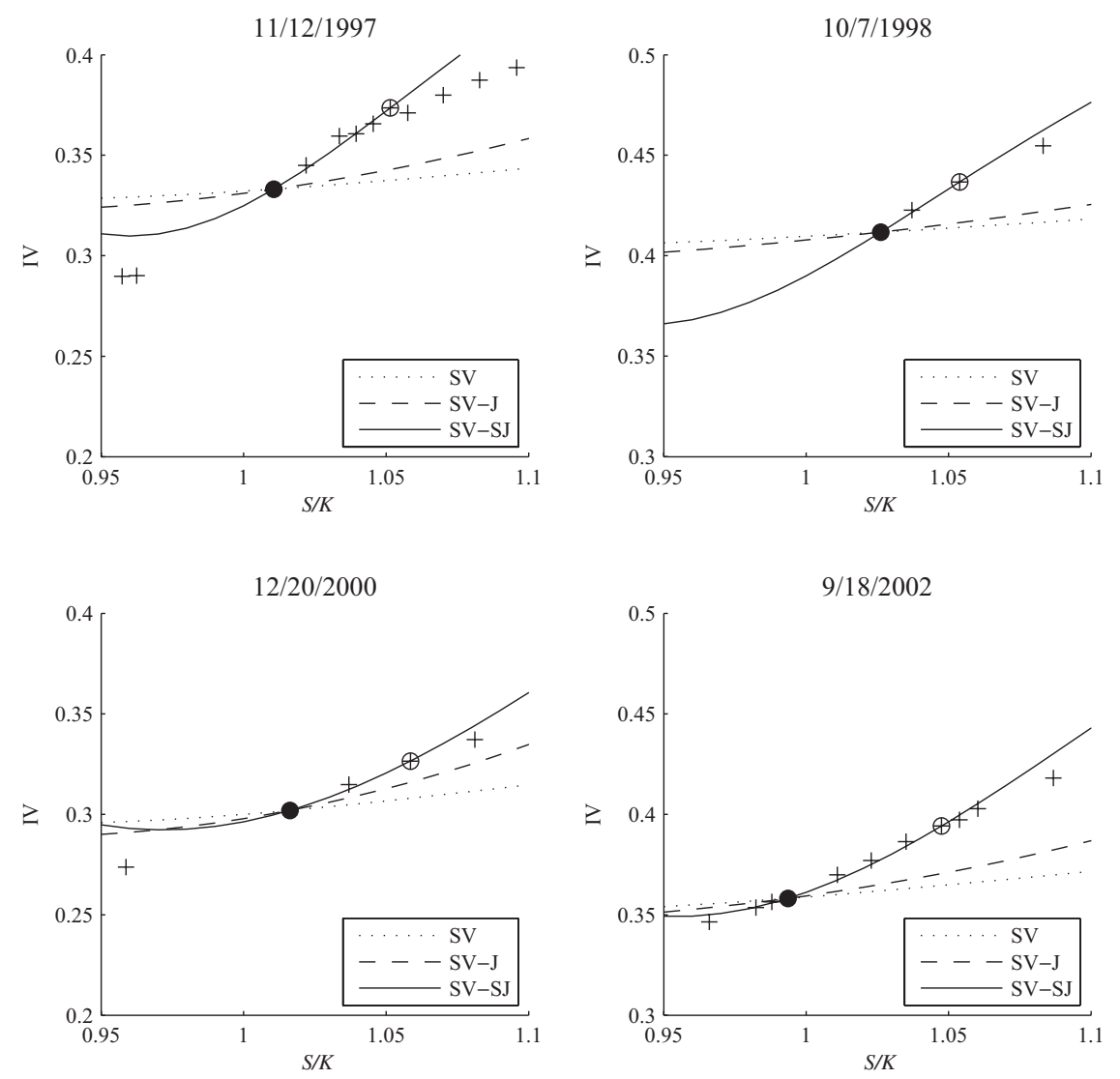

Note: We use the estimated parameters of the three models reported in table 2 to compute the fitted implied volatilities. The plus signs "+" represent the market implied volatilities. The fitted implied volatilities of the SV, SV-J, and SV-SJ models are represented by the dotted, dashed, and solid lines, respectively. "O" is the implied volatility of the near-the-money option that is fitted exactly by all three models, and " $\oplus$ " is the implied volatility of the out-of-the-money option that is fitted exactly by the SV-SJ model.

data set is $2.13 \%$, measured in units of implied volatility. This is smaller than the RMSEs of the SV and SV-J models, which are $3.35 \%$ and $2.73 \%$, respectively. Standard $t$-tests show that the RMSEs are significantly different from each other. For example, the $t$-statistic for a difference between the SV-J and SV-SJ models is 14.75. Despite the improvement in fitting the option prices, significant pricing errors remain as the RMSE of the SV-SJ model is still about twice the average bid-ask spread in our sample, which is $1.01 \%$ (with a standard deviation of $0.66 \%$ ), again in units of Black-Scholes implied volatility.

Figure 3 plots the market-implied volatilities of options with the shortest maturity, together with the fitted implied volatilities of the three alternative pricing models in four different dates of the sample. We find that the SV-SJ model does a much better job at pricing the cross-section of options than the other two models for these four days when the implied volatilities are high.

Having established that our model can effectively capture the time-series and cross-section properties of option prices, we now try to improve our understanding of the model. In particular, we want to understand the relative roles of the diffusive volatility and jump intensity in pricing options. Figure 4 shows the plots of implied volatility smiles at different maturities produced by our model, using the estimated parameters and different values of volatility and jump intensity. In the first two cases, the diffusive volatility, $\sqrt{V}$, is fixed at its sample average while the state variable for jump intensity, $Z$, is either at its sample average or 1 standard deviation above or below it. In the next two cases, the state variable for jump intensity, $Z$, is fixed at its sample average, while the diffusive volatility, $\sqrt{V}$, is either at its sample average or 1 standard deviation above or below it. The time to maturity is either 30 days or 90 days. We find that both volatility and jump intensity have an impact on the level of implied volatilities. Furthermore, the persistence in both risk components guarantees that their effects are felt at long horizons. But the two state variables have different impacts on the shape of the implied volatility smile. Jump intensity has a large impact on the prices of all short-term options but it affects out-of-the-money puts (high $S / K$ ) more than inthe-money puts (low $S / K$ ). The volatility has a larger impact on the prices of near-the-money options than those of away-from-the-money options. The longer the maturity, the flatter the volatility smiles, reflecting mean reversion in the volatility and jump intensity processes. The differential impact of volatility and jump intensity on 
Figure 4.-Volatility Smile of the SV-SJ Model
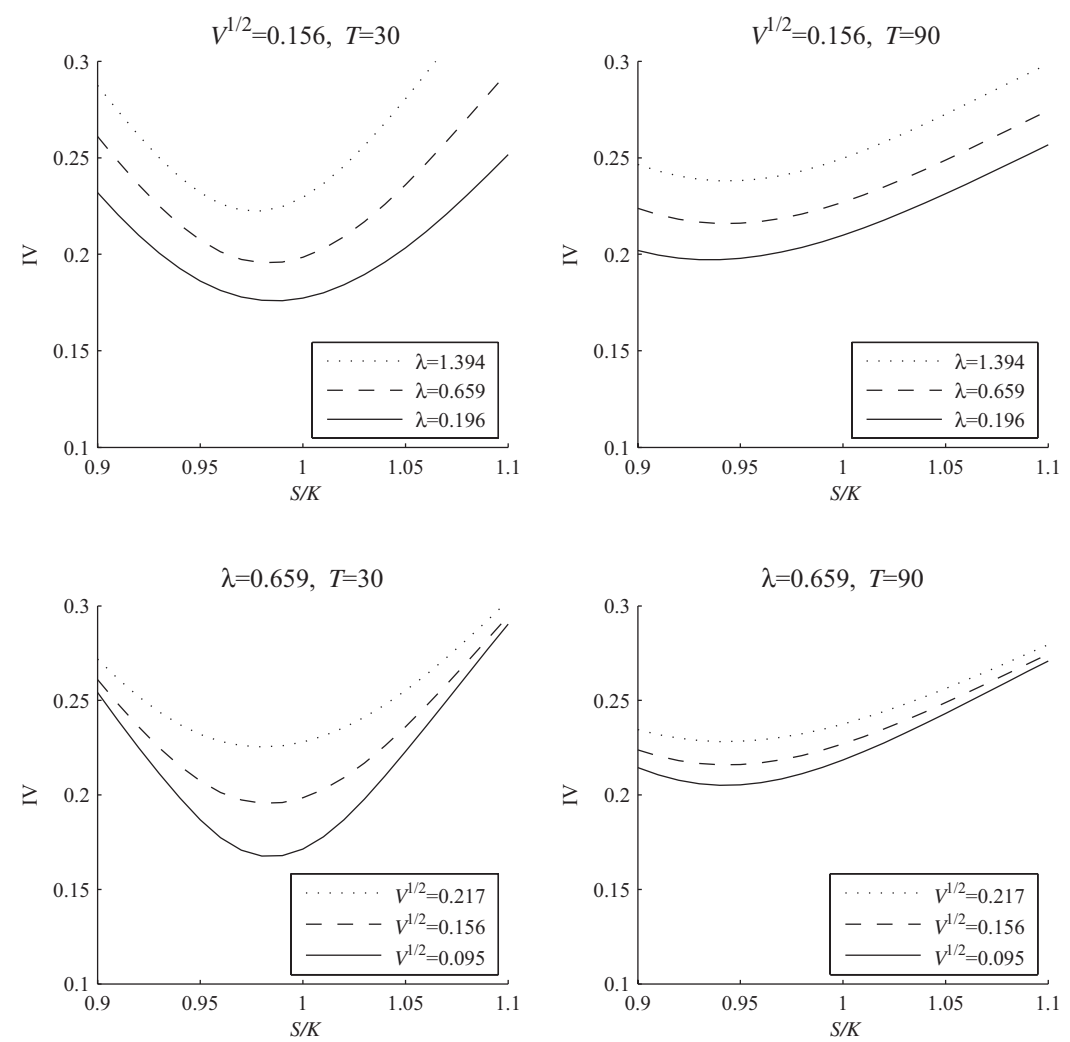

Note: The four panels show the plots of the Black-Scholes implied volatility smiles at different maturities produced by the SV-SJ model, using the estimated parameters reported in table 2 and for different values of volatility $(\sqrt{V})$ and jump intensity $(\lambda)$. In the top two panels, $\sqrt{V}$ is fixed at its sample average $(0.156)$ while $\lambda\left(=Z^{2}\right)$ is chosen so that $Z$ is at its sample average and that value plus or minus one standard deviation. In the bottom two panels, $\lambda$ is fixed so that $Z$ is at its sample average (0.812) while $\sqrt{V}$ is at its sample average and that value plus or minus one standard deviation. The maturities are 30 and 90 days for the left and right panels, respectively.

options of varying maturity and moneyness is what allows us to identify the two state variables in the estimation.

\section{Option-Implied Risks and the Equity Premium}

In this section we study the equilibrium equity premium implied by the parameter estimates and the implied state variables.

\section{A. The Equity Premium}

The estimate of $\gamma$ in table 2 for the SV-SJ model is 1.917 , which seems quite reasonable. In an economy without jumps and with constant volatility, Merton (1973) shows that the equity premium demanded by an investor who holds the stock market is equal to $\gamma$ times the market's variance. Since the realized volatility in our sample was $18.8 \%$, using the estimated risk aversion coefficient, we obtain an unconditional equity premium of $6.8 \%\left(1.917 \times 0.188^{2}\right)$. This premium approximately matches the historic average excess stock market return of between $4 \%$ and $9 \%$ (depending on the sample period) reported by Mehra and Prescott (2003). Note that we are studying the portfolio choice of an investor who derives utility from next period's wealth, not utility from lifetime consumption. In the latter case, it is well known from Mehra and Prescott (1985) and much subsequent work that a much higher level of risk aversion is needed to match the historic equity premium.

In what follows, we keep the horizon of the representative investor at 1 month, $T=1 / 12 .^{27}$ The choice of a short horizon abstracts away from hedging demands, making the interpretation of the results simpler. ${ }^{28}$ Given the relatively strong mean reversion in the risk processes, it is unlikely that horizons longer than one month would generate hedging demands strong enough to change the results. ${ }^{29}$

\footnotetext{
27 The results are robust to this choice of time horizon. We tried $T$ for up to ten years, and the main results do not change quantitatively. All the coefficients of $B$ and $C$ converge quickly to a limit as $T$ increases, and after one month, they are virtually constant. Moreover, these coefficients are small in magnitude. Their impact on the equity premium is correspondingly small. The component of the equity premium that involves $B$ and $C$ is generally negative with small magnitude (bounded by $2 \%$ and on average less than $0.5 \%$ ) in comparison to the average size of the equity premium, which is over $10 \%$. It is reasonable to say that $B$ and $C$ are not critical in determining the size and variation of the implied equity premium that we find.

${ }^{28}$ Note that we are considering preferences for terminal consumption. A given horizon in our model should be compared with the "duration" of utility in a model with intermediate consumption (which is necessarily less than the terminal date).

${ }^{29}$ Chacko and Viceira (2005) find that the hedging demands induced by stochastic volatility are tiny due to the strong mean reversion.
} 
Equation (13) gives us the equity premium as a function of the diffusive volatility and jump intensity. With the estimated parameters of the model, we can evaluate the coefficients of that function:

$$
\begin{aligned}
\phi= & 1.917 Y^{2}-0.008 Y-0.009 Y^{2} \\
& -0.022 Y Z+0.087 Z^{2} .
\end{aligned}
$$

Given the implied series of the diffusive volatility $Y$ and jump intensity $Z$, we can compute the average of the equity premium in our sample. This gives us an estimate of the unconditional equity premium of $11.8 \%$. Note that this is different from putting the average level of the implied series of the diffusive and jump risks in the above equation because of the nonlinearity of the equity premium in $Y$ and $Z$. Note also that this calculation does not match the average excess return of the S\&P 500 index in our sample, which is only $2 \%$. The reason is that we did not use stock returns in the calculation, only the measures of risk implied from option prices together with the estimated level of risk aversion.

Remember that the premium demanded by an investor with the same preferences in an economy without jumps and with constant volatility was $6.8 \%$. Therefore, the unconditional equity premium we computed with the risk inferred from option prices is more than $70 \%$ higher than the premium for realized risk. ${ }^{30}$

These findings have some bearing on the discussion of the equity premium puzzle first investigated by Mehra and Prescott (1985) and recently surveyed in Mehra and Prescott (2003). The equity premium puzzle is typically stated as the historic average stock market return far exceeding the compensation for its risk that would be required by an investor with a reasonable level of risk aversion. It should be noted that the literature on the equity premium puzzle usually measures risk by the covariance of stock market returns with aggregate consumption growth. However, none of our calculations involves consumption, and there is no way we can obtain the implied covariances between stock market returns and consumption growth from option prices. What we do show is that the risk premium demanded by an investor with utility for wealth living in an economy with the realized level of market volatility is only slightly more than half the premium demanded by the same investor when taking into account the risks assessed by option markets.

The puzzle is that the historic stock market premium of, say, $6 \%$, is much higher than the approximately $1 \%$ excess return warranted by the covariance of the stock market returns with consumption growth (for reasonable levels of

\footnotetext{
${ }^{30}$ The high equity premium obtained here may be caused by our choice of the sample period, which was much more volatile than other periods (1990-1996). It may also be partially driven by our specific option pricing model. As options may contain high premia, they can be translated into high premia in the stock returns. We thank an anonymous referee for pointing this out.
}

risk aversion). Our point is that the realized covariance of the stock market returns with consumption growth is likely to understate the true risk of the market by as much as the realized volatility understates the risk implicit in option prices. In our simple calculation above, we found that the ex ante risk premium almost doubles when we use the option implied risks instead of the realized volatility. If the same factor were to apply to the consumption-based risk measure, the equity premium puzzle would be considerably lessened. ${ }^{31}$

These results confirm a substantial peso problem when measuring the riskiness of the stock market with realized volatility. The risks investors perceive ex ante and that are therefore embedded in option prices far exceed the realized variation in stock market returns.

If investors price the stock market to deliver returns that compensate them for the perceived level of risk, the equity premium can easily be twice what is justifiable from the level of realized risk. This is the fundamental idea of Brown et al. (1995): ex post measured returns include a premium for some bad states of the world that investors deemed probable but did not materialize in the sample. Similarly, Rietz (1988) proposed a solution for the equity premium puzzle based on a very small probability (about 1\%) of a very large drop in consumption (25\%). That is not far from the risks perceived by investors in the option market. Barro (2006) extended the analysis of Rietz to show that rare events can explain a variety of asset pricing regularities. Goetzmann and Jorion (1999) provide empirical evidence that large jumps have occurred in a variety of countries in the twentieth century and that the United States was an outlier, with both few crashes and the highest realized average return.

Of course, this discussion only shifts the equity premium puzzle to a puzzlingly large difference between the level of perceived risk and the level of realized risk: the option market predicted a lot more market crashes than the number that actually occurred. For example, given the average jump size and average intensity estimated in tables 2 and 3, the stock market should experience market crashes with a magnitude of $-9.8 \%$ once every 1.26 years. This is obviously very different from the observed frequency and magnitude of stock market jumps. The interesting finding is that the puzzlingly high risks implicit in option markets match the puzzlingly high equity premium for very reasonable preferences.

\section{B. Time Variation in the Equity Premium}

The previous section discussed the unconditional equity premium. We now discuss the time variation in the equity premium. Figure 5 plots the time series of the risk premium

\footnotetext{
${ }^{31}$ Especially if we consider the estimates of the equity premium of around 3\% instead of 6\% that have been provided by Claus and Thomas (2001), Fama and French (2002), and Welch (2001).
} 
Figure 5.-EQUity Premium

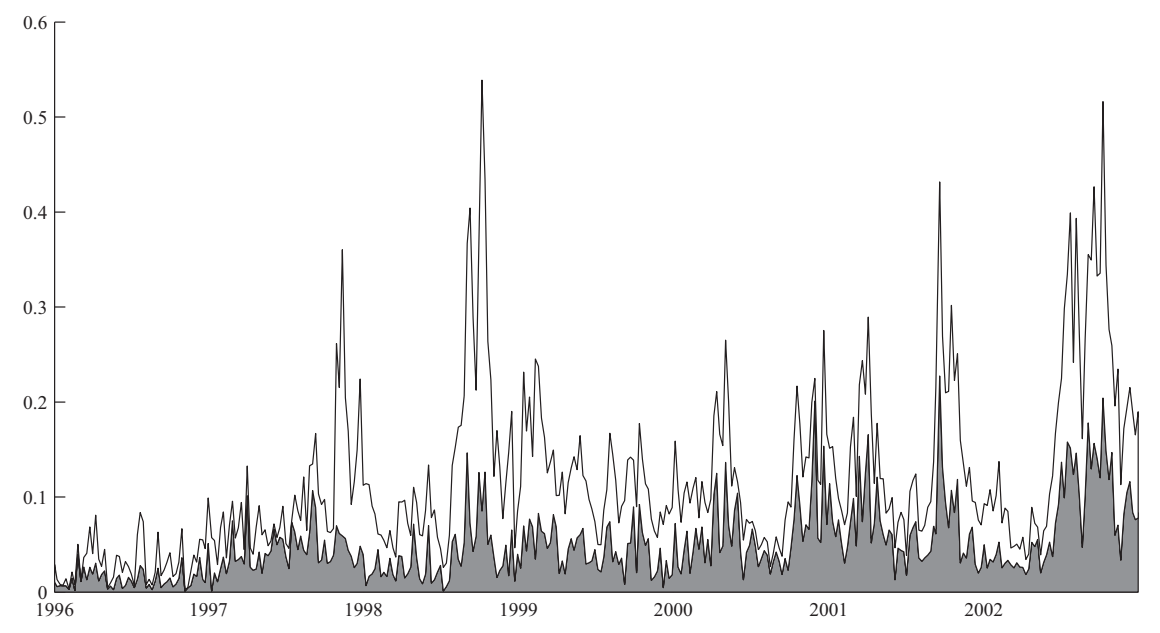

Note: The volatility component is represented by the gray area, while the jump component is represented by the white area.

demanded by the investor in our economy, shown in equation (35).

We decompose the premium in equation (35) into the compensation for the diffusive volatility, which encompasses the first three terms that depend only on $Y$, and the compensation for the jump risk involving the last term that depends only on $Z$. There is a small term that depends on the product of $Y$ and $Z$, which shows up in the total premium but that we do not assign to the components.

The plot of the time series of the equity premium shows high variability. Its standard deviation in our sample is $8.9 \%$, roughly three-quarters the unconditional premium of $11.8 \%$. The premium ranges from $0.3 \%$ to $54.9 \%$. Furthermore, the first-order serial correlation (at monthly frequency) of the premium is 0.82 , which shows persistence but is far from a unit root. However, we should note that all the first ten serial correlations are positive and add up to 5.044. There is therefore memory in the equity premium that is not easily captured by a simple autoregression.

The jump component is on average $6.9 \%$, or a bit more than half of the total equity premium. Its standard deviation is of the same order of magnitude: $6.2 \%$. The jump premium varies between 0 and $45.4 \%$ and represents at times nearly the entire equity premium. The jump component of the equity premium is also more persistent than the volatility component, with first-order serial correlations of 0.80 and 0.63 , respectively. The sum of the first 10 serial correlations is also higher, 4.88 versus 4.37 .

These numbers obtain under very strong assumptions. A conditional equity premium that is as strongly time varying as the one reported here implies an economy in which the investors have significant ability to time the market. Our model likely overstates the variability of the equity premium due to in-sample overfitting and the very specific nature of our parametric model.

However, note that the recent literature on stock market predictability implies that all the variation in market valu- ation multiples corresponds to changes in expected excess returns, that is, the equity premium, and none corresponds to news about future dividend growth. Cochrane (2008) estimates that the standard deviation of market expected returns is about 5 percentage points (the same magnitude as the premium itself) using only the dividend yield as a predicting variable. When more variables are used (and many have been identified in the literature), the volatility of the equity premium increases. This is still an order of magnitude less than the equity premium variability we estimate, but it is remarkable that such volatility is found from regressions with ex post returns. Using ex ante information as we do should lead to an equity premium that is even more variable.

\section{Forecasting Stock Returns with the Implied Equity Premium}

As discussed above, the model implied equity premium varies over time. We next investigate if this variation is reflected in the realized stock returns. In particular, we conduct the following predictive regression analysis: ${ }^{32}$

$$
R_{t, t+k}=\alpha+\beta X_{t}+\epsilon_{t, t+k},
$$

where $R_{t, t+k}$ is the annualized average stock returns from week $t$ to week $t+k$, and $X_{t}$ is the predictive variable observed at $t$. We consider values of $k$ to be one, four, eight, and thirteen, corresponding to horizons of one week, one month, two months, and three months. We choose the predictor, $X_{t}$, to be the SV-SJ model implied state variables $Y_{t}$ and $Z_{t}$, and the equity premium $\phi_{t}$. If our model is right, then when using $\phi_{t}$ to forecast stock returns, a significant positive coefficient estimate of $\beta$ is expected. In fact, this coefficient should be close to 1 . To increase statistical

\footnotetext{
${ }^{32}$ Note that we have used the full sample to obtain the model-implied state variables and equity premium. This introduces some forward-looking bias in our predictive regressions.
} 
Table 4.-Predicting Stock Returns Using the Option IMPLIED EQUITY PREMIUM

\begin{tabular}{|c|c|c|c|c|}
\hline \multicolumn{5}{|c|}{1 Week } \\
\hline$Y$ & $\begin{array}{c}1.438 \\
(1.026)\end{array}$ & & $\begin{array}{c}0.008 \\
(0.006)\end{array}$ & \\
\hline$Z$ & & $\begin{array}{c}0.386 \\
(1.566)\end{array}$ & $\begin{array}{c}0.386 \\
(1.483)\end{array}$ & \\
\hline$\phi$ & & & & $\begin{array}{c}2.139 \\
(1.863)\end{array}$ \\
\hline$R^{2}$ & 0.004 & 0.011 & 0.011 & 0.020 \\
\hline \multicolumn{5}{|c|}{1 Month } \\
\hline$Y$ & $\begin{array}{c}0.850 \\
(0.965)\end{array}$ & & $\begin{array}{c}-0.300 \\
(-0.333)\end{array}$ & \\
\hline$Z$ & & $\begin{array}{c}0.279 \\
(1.746)\end{array}$ & $\begin{array}{c}0.310 \\
(1.791)\end{array}$ & \\
\hline$\phi$ & & & & $\begin{array}{c}1.456 \\
(2.126)\end{array}$ \\
\hline$R^{2}$ & 0.007 & 0.026 & 0.026 & 0.041 \\
\hline \multicolumn{5}{|c|}{2 Months } \\
\hline$Y$ & $\begin{array}{c}0.789 \\
(1.197)\end{array}$ & & $\begin{array}{c}-0.547 \\
(-0.722)\end{array}$ & \\
\hline$Z$ & & $\begin{array}{c}0.305 \\
(2.387)\end{array}$ & $\begin{array}{c}0.360 \\
(2.434)\end{array}$ & \\
\hline$\phi$ & & & & $\begin{array}{c}1.360 \\
(2.655)\end{array}$ \\
\hline$R^{2}$ & 0.012 & 0.065 & 0.068 & 0.076 \\
\hline \multicolumn{5}{|c|}{3 Months } \\
\hline$Y$ & $\begin{array}{c}0.158 \\
(0.251)\end{array}$ & & $\begin{array}{c}-1.154 \\
(-1.689)\end{array}$ & \\
\hline$Z$ & & $\begin{array}{c}0.250 \\
(1.941)\end{array}$ & $\begin{array}{c}0.362 \\
(2.676)\end{array}$ & \\
\hline$\phi$ & & & & $\begin{array}{c}1.001 \\
(1.868)\end{array}$ \\
\hline$R^{2}$ & 0.001 & 0.074 & 0.101 & 0.066 \\
\hline
\end{tabular}
parentheses.

power, we use overlapping samples for multiperiod regressions. To adjust for heteroskedasticity and serial correlation in the regression residuals, the standard errors are calculated using the Newey-West method.

Table 4 reports the results of the predictive regression. ${ }^{33}$ From the first column, $Y$, the implied state variable for the diffusive volatility is positively related to future stock returns but is not significant. From the second column, $Z$, the implied state variable for the jump intensity is also positively related to future stock returns. More interesting, the $t$-statistics for $Z$ are much higher than those for $Y$. The $R^{2} \mathrm{~s}$ are also much larger. When both $Y$ and $Z$ are used in column 3, $Y$ remains insignificant, while $Z$ is significant for horizons of two and three months. As seen in the fourth column, the estimated regression coefficient on $\phi$ decreases from 2.139 for one-week returns to 1.001 for three-month returns, and it is always significant at the $90 \%$ confidence level. The

\footnotetext{
${ }^{33}$ Related evidence is provided by Doran, Peterson, and Tarrant (2006), who show that the put volatility skew has strong predictive power in forecasting short-term market declines. This is consistent with our model, where the volatility skew is strongly correlated with jump risk and its corresponding risk premium.
}

$t$-statistic is highest for two-month returns. The $R^{2}$ initially increases from 0.020 for one-week returns to 0.076 for two-month returns, and then decreases to 0.066 for threemonth returns. These results suggest that the model-implied equity premium has significant predictive power of future stock returns for horizon up to three months. The predictive power seems to be coming from stochastic jump intensity.

Of course, this analysis has several limitations. First, we have a limited sample to run the predictive regressions. It is remarkable that we obtain significant estimates at all. Second, the problem of relating the equity premium from option prices to the realized equity returns is precisely the peso problem. We argue that options capture a risk that is perceived as likely by the investors even if it does not materialize in the realized returns. This should bias the estimates of our regressions.

\section{Relation between the Implied Equity Premium and Financial Crisis Variables}

We have argued that the implied equity premium may capture the stock market crash risk, which may be caused by liquidity shocks and crisis in the financial system. It is therefore interesting to examine the relation between the implied equity premium with financial variables that are often regarded as correlated with financial crisis. We consider three such variables for the period of 1996-2002: the three-month T-bill rate (Tbill); the commercial paper spread (CPS), which is the difference between the rates of threemonth financial commercial paper and the three-month T-bill; and the high-yield spread (HYS), which is the difference between the average yield of the Merrill Lynch high-yield corporate bond index and the yield of the tenyear Treasury bond. ${ }^{34}$ The CPS is only available since 1997. All data are obtained from Datastream.

The top panel of table 5 reports summary statistics for the three variables. We examine the relation between our model implied equity premium (and state variables) and the financial crisis variables by considering the following regression:

$$
\Delta Y_{t}=\alpha+\beta \Delta X_{t}+\epsilon_{t},
$$

where the dependent variable $Y_{t}$ is the Tbill, CPS, or HYS, and $X_{t}$ is $V_{t}, \lambda_{t}$, or $\phi_{t}$.

The second panel of table 5 reports the results of the above regressions. The change in Tbill is negatively related to changes in $V, \lambda$, and $\phi$ but only significantly so for $\lambda$ and $\phi$. In contrast, the changes in CPS and HYS are positively and significantly related to changes in $V, \lambda$, and $\phi$. This implies that when diffusive volatility and jump intensity increase (decrease) the commercial paper spread and the

${ }^{34}$ The Fed cut interest rates after the stock market crash of October 1987, the Russian financial crisis of 1998, the collapse of technology stocks in 2000, and the 2007 subprime mortgage debacle. During credit crisis, investors refuse to roll over commercial papers and instead turn to other short-term safe havens, such as the T-bills. 
TABle 5.-Financial CRisis Variables

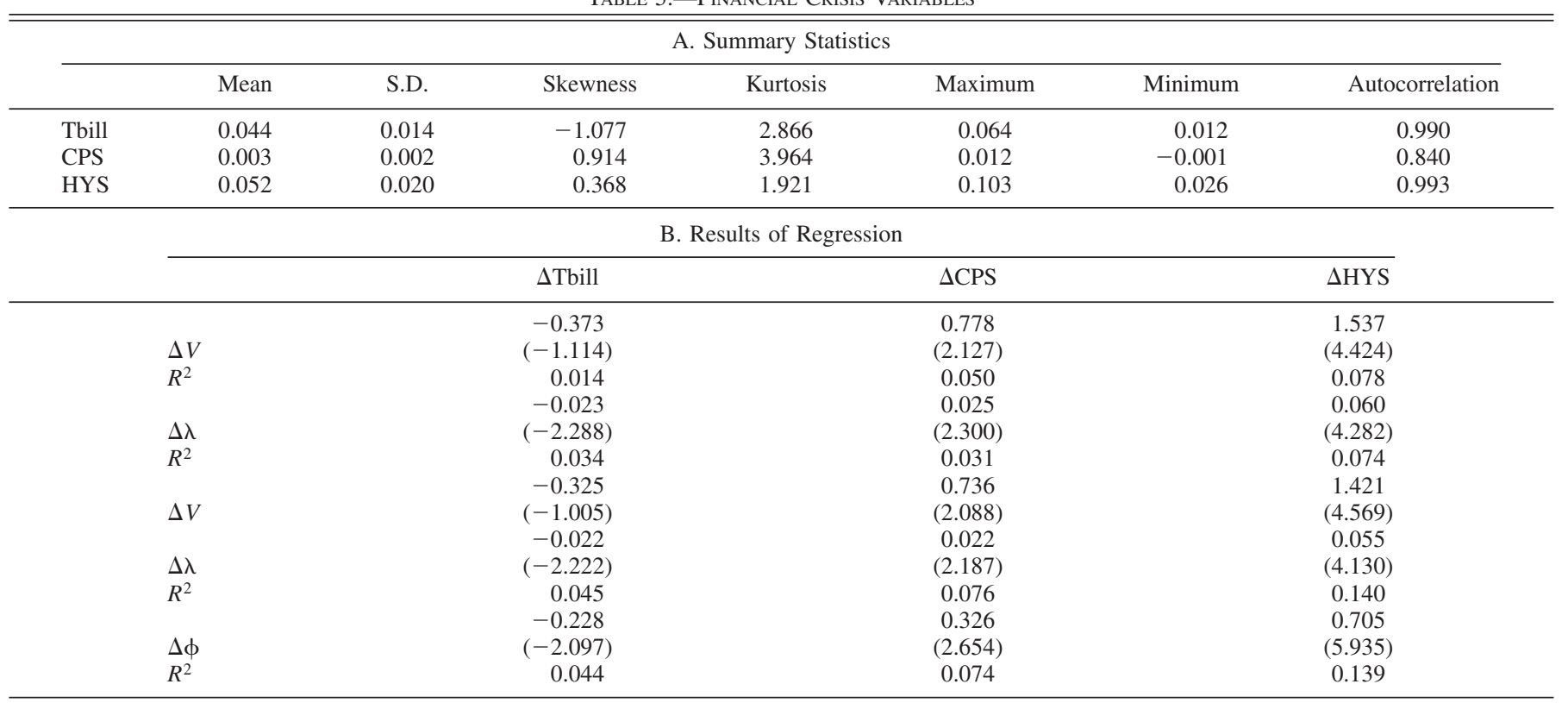

Note: We use the Newey-West method to compute standard errors. $t$-statistics are reported in parentheses.

high-yield spread tend to widen (narrow). The $R^{2}$ for the CPS and HYS regressions are generally higher than those for the Tbill regressions. These results indirectly suggest that our implied equity premium captures a risk perceived by investors that financial markets may collapse.

More informally, we can look at the largest crisis in our sample. In the fall of 1998, following the Asian crisis, at the time of the Russian default and the LTCM blowup, the option-implied equity premium rose from $2 \%$ to $55 \%$ per year within a couple of months. With such a dramatic increase in the equity premium, we should expect a sharp drop in stock prices. The stock market decline during that period was on the order of $20 \%$. The sign of this change is consistent with a large increase of the equity premium but perhaps quantitatively smaller than what the actual change in the equity premium would justify. Note that here the peso problem becomes very apparent. In fall 1998, fear was widespread of a financial crisis that might lead to bank defaults. Had that occurred, the stock market would likely have fallen much more. As it turned out, the Fed was able to engineer a bailout of LTCM, the banking crisis was avoided, and the stock market did not crash.

\section{Conclusion}

We imply the time series of diffusive volatility and jump intensity from S\&P 500 index options. These are the ex ante risks in the stock market assessed by option investors. We find that both components of risk vary substantially over time, are quite persistent, and correlate with each other and with the stock index. Using a simple general equilibrium model with a representative investor, we translate the implied measures of ex ante risk into an ex ante risk premium.
We find that the average premium that compensates the investor for the risks implicit in option prices, $11.8 \%$, is about $40 \%$ higher than the premium required to compensate the same investor for the realized volatility in stock market returns, $6.8 \%$. These results support the peso explanation advanced by Rietz (1988), Brown et al. (1995), and Barro (2006) for the equity premium puzzle of Mehra and Prescott (1985). We also find that the ex ante equity premium is highly volatile, taking values between $0.3 \%$ and $54.9 \%$, with the component of the premium that corresponds to the jump risk varying between $0 . \%$ and $45.4 \%$. The optionimplied equity premium is shown to forecast subsequent stock returns.

In summary, we are able to partially explain the equity premium puzzle by using measures of risk implied from option prices that far exceed measures of realized risk. We are still left with a puzzle: like Aesop's boy, the option markets cry wolf a lot more often than the wolf actually shows up. However, it is interesting that we can link, using reasonable levels of risk aversion, the puzzlingly high equity premium observed historically with puzzlingly high risks implicit in option markets.

\section{REFERENCES}

Ahn, Dong-Hyun, Robert F. Dittmar, and A. Ronald Gallant, "Quadratic Term Structure Models: Theory and Evidence," Review of Financial Studies 15:1 (2002), 243-288.

Ait-Sahalia, Yacine, Yobo Wang, and Francis Yared, "Do Option Markets Correctly Price the Probabilities of Movement of the Underlying Asset?" Journal of Econometrics 102:1 (2001), 67-110.

Alizadeh, Sassan, Michael W. Brandt, and Francis X. Diebold, "RangeBased Estimation of Stochastic Volatility Models," Journal of Finance 57:3 (2002), 1047-1091. 
Anderson, Torben, Luca Benzoni, and Jesper Lund, "An Empirical Investigation of Continuous-Time Equity Return Models," Journal of Finance 57:3 (2002), 1239-1284.

Bakshi, Gurdip, Charles Cao, and Zhiwu Chen, "Empirical Performance of Alternative Option Pricing Models," Journal of Finance 52:5 (1997), 2003-2049.

Ball, Clifford A., and Antonio Roma, "Stochastic Volatility Option Pricing," Journal of Financial and Quantitative Analysis 29:4 (1994), 589-667.

Ball, Clifford A., and Walter N. Torous, "A Simplified Jump Process for Common Stock Returns," Journal of Financial and Quantitative Analysis 18:1 (1983), 53-65.

Barro, Robert J., "Rare Disasters and Asset Markets in the Twentieth Century," Quarterly Journal of Economics 121:3 (2006), 823-866.

Bates, David, "Jumps and Stochastic Volatility: Exchange Rate Processes Implicit in Deutsche Mark Options," Review of Financial Studies 9:1 (1996), 69-107.

"Post-'87 Crash Fears in the S\&P 500 Futures Option Market," Journal of Econometrics 94:1 (2000), 181-238.

"The Market for Crash Risk," University of Iowa working paper (2001).

Black, Fischer, and Myron Scholes, "The Pricing of Options and Corporate Liabilities," Journal of Political Economy 81:3 (1973), 637 654.

Bliss, Robert R., and Nikolaos Panigirtzoglou, "Option-Implied Risk Aversion Estimates," Journal of Finance 59:1 (2004), 407-446.

Broadie, Mark, Mike Chernov, and Michael Johannes, "Model Specification and Risk Premia: Evidence from S\&P 500 Futures Options Market," Journal of Finance 62:3 (2007), 1453-1490.

Brown, Stephen J., William N. Goetzmann, and Stephen A. Ross, "Survival," Journal of Finance 50:3 (1995), 853-873.

Chacko, George, and Luis Viceira, "Spectral GMM Estimation of Continuous-Time Processes," Journal of Econometrics 116:1 (2003), 259-292.

"Dynamic Consumption and Portfolio Choice with Stochastic Volatility in Incomplete Markets," Review of Financial Studies 18:4 (2005), 1369-1402.

Chen, Li, Damir Filipovic, and H. Vicent Poor, "Quadratic Term Structure Models for Risk-Free and Defaultable Rates," Mathematical Finance 14:4 (2004), 515-536.

Cheng, Peng, and Olivier Scaillet, "Linear-Quadratic Jump-Diffusion Modelling," Mathematical Finance 17:4 (2007), 575-598.

Chernov, Mikhael, A. Ronald Gallant, Eric Ghysels, and George Tauchen, "Alternative Models for Stock Price Dynamics," Journal of Econometrics 116:1 (2002), 225-257.

Chernov, Mikhael, and Eric Ghysels, "A Study towards a Unified Approach to the Joint Estimation of Objective and Risk Neutral Measures for the Purpose of Options Valuation," Journal of Financial Economics 56:3 (2000), 407-458.

Claus, James, and Jacob Thomas, "Equity Premia as Low as Three Percent? Evidence from Analysts Earnings Forecasts for Domestic and International Stocks," Journal of Finance 56:5 (2001), 16291666.

Cochrane, John, "The Dog That Did Not Bark: A Defense of Return Predictability," Review of Financial Studies 21 (2008), 1533-1575.

Coval, Joshua D., and Tyler Shumway, "Expected Option Returns," Journal of Finance 56:3 (2001), 983-1009.

Doran, James, David Peterson, and Brian Tarrant, "Is There Information in the Volatility Skew?" Florida State University working paper (2006).

Driessen, Joost, and Pascal Maenhout, "A Portfolio Perspective on Option Pricing Anomalies," INSEAD working paper (2003).

Duffee, Gregory R., "Term Premia and Interest Rate Forecasts in Affine Models," Journal of Finance 57:1 (2002), 405-443.

Duffie, Darrell, Jun Pan, and Kenneth Singleton, "Transform Analysis and Asset Pricing for Affine Jump-Diffusions," Econometrica 68:6 (2000), 1343-1376.

Engle, Robert F., and Gary G. J. Lee, "A Long-Run and Short-Run Component Model of Stock Return Volatility," in Robert F. Engle and Halbert White (Eds.), Cointegration, Causality and Forecasting: A Festschrift in Honour of Clive W. J. Granger (New York: Oxford University Press, 1999).

Engle, Robert F., and Joshua Rosenberg, "Empirical Pricing Kernels," Journal of Financial Economics 64:3 (2002), 341-372.

Eraker, Bjorn, "Do Stock Prices and Volatility Jump? Reconciling Evidence from Spot and Option Prices," Journal of Finance 59:3 (2004), 1367-1403.

Eraker, Bjorn, Michael S. Johannes, and Nicholas Polson, "The Impact of Jumps in Returns and Volatility," Journal of Finance 58:3 (2003), 1269-1300.

Fama, Eugene, and Kenneth French, "The Equity Premium," Journal of Finance 57:2 (2002), 637-659.

Ghysels, Eric, Pedro Santa-Clara, and Rossen Valkanov, "There Is a Risk-Return Tradeoff after All," Journal of Financial Economics 76:3 (2005), 509-548.

Goetzmann, William N., and Philippe Jorion, "Global Stock Markets in the Twentieth Century," Journal of Finance 54:3 (1999), 953-980.

Hamilton, James D., Time Series Analysis (Princeton, NJ: Princeton University Press, 1994).

Heston, Steven L., "A Closed-Form Solution for Options with Stochastic Volatility with Applications to Bond and Currency Options," Review of Financial Studies 6:2 (1993), 327-343.

Ingersoll, Jonathan E., Theory of Financial Decision Making (New York: Rowman \& Littlefield, 1987).

Jackwerth, Jans C., "Recovering Risk Aversion from Options Prices and Realized Returns," Review of Financial Studies 13:2 (2000), 433451.

Jorion, Philippe, "On Jump Processes in the Foreign Exchange and Stock Markets," Review of Financial Studies 1:4 (1988), 427-445.

Leippold, Markus, and Liuren Wu, "Asset Pricing under the Quadratic Class," Journal of Financial and Quantitative Analysis 37:2 (2002), 271-295.

Lewis, Alan L., Option Valuation under Stochastic Volatility (Newport Beach, CA: Finance Press, 2000).

Liu, Jun, and Jun Pan, "Dynamic Derivative Strategies," Journal of Financial Economics 69:3 (2003), 401-430.

Mehra, Rajnish, and Edward C. Prescott, "The Equity Premium: A Puzzle," Journal of Monetary Economics 15 (1985), 145-161.

"The Equity Premium in Retrospect," in George M. Constantinedes, Milton Harris, and René M. Stulz (Eds.), Handbook of the Economics of Finance (Amsterdam: Elsevier, 2003).

Merton, Robert C., "An Intertemporal Capital Asset Pricing Model," Econometrica 41:5 (1973), 867-887.

"Option Pricing When the Underlying Stock Returns Are Discontinuous," Journal of Financial Economics 4:1 (1976), 125-144. "On Estimating the Expected Return on the Market: An Exploratory Investigation," Journal of Financial Economics 8:4 (1980), 323-361.

Naik, Vasant, and Moon Lee, "General Equilibrium Pricing of Options on the Market Portfolio with Discontinuous Returns," Review of Financial Studies 3:4 (1990), 493-521.

Pan, Jun, "The Jump-Risk Premia Implicit in Options: Evidence from an Integrated Time-Series Study," Journal of Financial Economics 63:1 (2002), 3-50.

Protter, Philip, Stochastic Integration and Differential Equations (New York: Springer Verlag, 1990).

Rietz, Thomas A., "The Equity Premium Puzzle: A Solution?" Journal of Monetary Economics 22:1 (1988), 117-131.

Santa-Clara, Pedro, and Alessio Saretto, "The Risk and Return of Option Strategies," UCLA working paper (2004).

Schobel, Rainer, and Jianwei Zhu, "Stochastic Volatility with an Ornstein Uhlenbeck Process: An Extension," European Finance Review 3:1 (1999), 23-46.

Stein, Elias M., and Jeremy C. Stein, "Stock Price Distributions with Stochastic Volatility: An Analytic Approach," Review of Financial Studies 4:4 (1991), 727-752.

Welch, Ivo, "The Equity Premium Consensus Forecast Revisited," Yale University working paper (2001). 